\title{
p53 isoforms regulate astrocyte-mediated neuroprotection and neurodegeneration
}

\author{
C Turnquist ${ }^{1}$, I Horikawa ${ }^{1}$, E Foran ${ }^{2}$, EO Major ${ }^{3}$, B Vojtesek ${ }^{4}$, DP Lane ${ }^{5}$, X Lu ${ }^{6}$, BT Harris ${ }^{*, 7,8,9}$ and CC Harris ${ }^{*, 1,9}$
}

Bidirectional interactions between astrocytes and neurons have physiological roles in the central nervous system and an altered state or dysfunction of such interactions may be associated with neurodegenerative diseases, such as Alzheimer's disease (AD) and amyotrophic lateral sclerosis (ALS). Astrocytes exert structural, metabolic and functional effects on neurons, which can be either neurotoxic or neuroprotective. Their neurotoxic effect is mediated via the senescence-associated secretory phenotype (SASP) involving pro-inflammatory cytokines (e.g., IL-6), while their neuroprotective effect is attributed to neurotrophic growth factors (e.g., NGF). We here demonstrate that the p53 isoforms $\Delta 133 p 53$ and p53 $\beta$ are expressed in astrocytes and regulate their toxic and protective effects on neurons. Primary human astrocytes undergoing cellular senescence upon serial passaging in vitro showed diminished expression of $\Delta 133 \mathrm{p} 53$ and increased p53 $\beta$, which were attributed to the autophagic degradation and the SRSF3-mediated alternative RNA splicing, respectively. Early-passage astrocytes with $\Delta 133 p 53$ knockdown or p53 $\beta$ overexpression were induced to show SASP and to exert neurotoxicity in co-culture with neurons. Restored expression of $\Delta 133 p 53$ in near-senescent, otherwise neurotoxic astrocytes conferred them with neuroprotective activity through repression of SASP and induction of neurotrophic growth factors. Brain tissues from AD and ALS patients possessed increased numbers of senescent astrocytes and, like senescent astrocytes in vitro, showed decreased $\Delta 133 p 53$ and increased p53 $\beta$ expression, supporting that our in vitro findings recapitulate in vivo pathology of these neurodegenerative diseases. Our finding that $\Delta 133 p 53$ enhances the neuroprotective function of aged and senescent astrocytes suggests that the p53 isoforms and their regulatory mechanisms are potential targets for therapeutic intervention in neurodegenerative diseases.

Cell Death and Differentiation (2016) 23, 1515-1528; doi:10.1038/cdd.2016.37; published online 22 April 2016

Brain aging and neurodegenerative diseases remain major medical challenges of the twenty-first century. Alzheimer's disease (AD), the most common form of dementia, affects about 36 million people worldwide and is estimated to have cost $\$ 604$ billion in 2010 alone. ${ }^{1}$ Amyotrophic lateral sclerosis (ALS) is characterized by the loss of upper and lower motor neurons and life expectancy is $2-5$ years following diagnosis. It is estimated to affect about 500000 individuals in the USA alone and costs are \$256-\$433 million per year. ${ }^{2}$ Despite continued scientific effort, few effective therapies exist for these neurodegenerative diseases.

Astrocytes are the most abundant cell type in the brain and have key roles in providing structural, functional, and metabolic support to neurons. ${ }^{3}$ Although AD and ALS possess different etiologies, a commonality between these two diseases is the role of astrocytes in neurodegeneration. ${ }^{4,5}$ Dysfunctional neuron-astrocyte crosstalk is known to be a central feature of neurodegenerative diseases. ${ }^{6,7}$ Astrocytes exert both toxic and protective effects on neurons in a contextdependent manner. The neurotoxic effects of astrocytes are mediated in part through pro-inflammatory cytokines, such as interleukin-6 (IL-6). Overproduction of these factors is associated with human neurodegeneration ${ }^{8}$ and murine models. ${ }^{9}$ The neuroprotective effects of astrocytes are mediated through nerve growth factor (NGF) and insulin-like growth factor-1 (IGF-1), ${ }^{10}$ which are deficient in neurodegenerative diseases $^{11,12}$ and, to a lesser extent, during physiological brain aging. ${ }^{13}$ Alexander disease, a rare astrocyte disease

\footnotetext{
${ }^{1}$ Laboratory of Human Carcinogenesis, Center for Cancer Research, National Cancer Institute, National Institutes of Health, Bethesda, MD 20892, USA; ${ }^{2}$ Neurogenetics Branch, National Institute of Neurological Disorders and Stroke, National Institutes of Health, Bethesda, MD 20892, USA; ${ }^{3}$ Laboratory of Molecular Medicine and Neuroscience, National Institute of Neurological Disorders and Stroke, National Institutes of Health, Bethesda, MD, USA; ${ }^{4}$ Regional Centre for Applied and Molecular Oncology, Masaryk Memorial Cancer Institute, Brno 65653, Czech Republic; ${ }^{5}$ 553 Laboratory, Biomedical Sciences Institutes (A*STAR), Singapore 138648, Singapore; ${ }^{6}$ Ludwig Institute for Cancer Research, Nuffield Department of Clinical Medicine, University of Oxford, Oxford OX3 7DQ, UK; ${ }^{7}$ Department of Neurology, Georgetown University Medical Center, Washington DC 20007, USA and ${ }^{8}$ Department of Pathology, Georgetown University Medical Center, Washington DC 20007, USA

${ }^{*}$ Corresponding author: BT Harris, Departments of Neurology and Pathology, Georgetown University Medical Center, 4000 Reservoir Road, NW, Building D, Room 207, Washington, DC 20057, USA. Tel: 202687 5345; Fax: 202687 7378; E-mail: Bth@ georgetown.edu

or CC Harris, Laboratory of Human Carcinogenesis, Center for Cancer Research, National Cancer Institute, National Institutes of Health, 37 Convent Drive, Room 3068 A, Bethesda, MD 20892, USA. Tel: 301496 2048; Fax: 301496 0497; E-mail: Curtis_Harris@nih.gov

${ }^{9}$ These authors contributed equally to this work.

Abbreviations: AD, Alzheimer's disease; ALS, amyotrophic lateral sclerosis; DAPI, 4',6-diamidino-2-phenylindole; EAAT2, excitatory amino acid transporter-2; GFAP, glial fibrillary acidic protein; IGF-1, insulin-like growth factor-1; IL, interleukin; IL-6-NAb, IL-6 neutralizing antibody; iPSC, induced pluripotent stem cell; NGF, nerve growth factor; NGF-NAb, NGF neutralizing antibody; NOS2, nitric oxide synthase 2; SA- $\beta$-Gal, senescence-associated $\beta$-galactosidase; SASP, senescence-associated secretory phenotype; $\gamma \mathrm{H} 2 \mathrm{AX}$, phosphorylated $\mathrm{H} 2 \mathrm{AX}$

Received 23.10.15; revised 11.3.16; accepted 19.3.16; Edited by N Bazan; published online 22.4.2016
} 
involving a mutation in the glial fibrillary acidic protein (GFAP), results in neurodegeneration. ${ }^{14}$ Riluzole, a Food and Drug Administration-approved drug for ALS, targets a glutamate transporter, EAAT2, in astrocytes. ${ }^{15}$ Thus, development of therapies targeting astrocytes is a subject of intense research. ${ }^{4,5}$

Cellular senescence is characterized by proliferation arrest after extensive cell divisions or upon cellular stresses. The characteristics of cellular senescence include enlarged cell morphology, upregulation of cell cycle inhibitors $\mathrm{p} 16^{\text {INK4 }}$ (ref. 16) and p21 ${ }^{\text {WAF1 } 1,{ }^{17}}$ senescence-associated $\beta$-galactosidase (SA- $\beta$-Gal) activity, ${ }^{18}$ elevated nitric oxide synthase 2 (NOS2) associated with oxidative stress, ${ }^{19,20}$ and DNA double-strand breaks marked by phosphorylated H2AX $(\gamma \mathrm{H} 2 \mathrm{AX}) .{ }^{21}$ Senescent cells secrete various cytokines, growth factors, and proteases that alter tissue microenvironment, which is collectively termed the senescence-associated secretory phenotype (SASP). ${ }^{22}$ A major outcome of SASP is tissue inflammation and degeneration mainly through the activity of pro-inflammatory cytokines. In addition to these unfavorable effects, senescent cells and SASP can have beneficial roles in wound healing ${ }^{23,24}$ and embryonic development. ${ }^{25-27}$

A main regulator of cellular stress responses and senescence is the TP53 gene. ${ }^{28}$ The human TP53 encodes, in addition to full-length $\mathrm{p} 53$ protein, at least 12 natural protein isoforms through alternative mRNA splicing or alternative promoter usage. ${ }^{29} \Delta 133 p 53$, an $\mathrm{N}$-terminal truncated isoform, functions as a negative regulator of cellular senescence by dominant-negatively inhibiting full-length p53. ${ }^{30,31} \mathrm{p} 53 \beta$, a C-terminal modified isoform, functions as a co-activator of full-length p53 to promote senescence. ${ }^{30,31}$

In this study, we report that $\Delta 133 p 53$ and $p 53 \beta$ regulate the neuroprotective and neurotoxic functions of human astrocytes. Using a neuron-astrocyte co-culture system, we demonstrate that downregulation of $\triangle 133 p 53$ or upregulation of p53 $\beta$ in astrocytes promotes SASP and non-cell autonomous neurotoxicity. Furthermore, reconstituted expression of $\triangle 133 p 53$ in neurotoxic astrocytes prevents SASP and reverses them to neuroprotective astrocytes.

\section{Results}

Astrocytes express p53 isoforms, $\Delta 133 p 53$ and p53 $\beta$, and their expression is regulated during cellular senescence. To examine whether p53 isoforms $\Delta 133 p 53$ and p53 $\beta$ are expressed in the human brain, immunofluorescence staining using $\Delta 133 p 53$-specific antibody MAP4 and p53 $\beta$-specific antibody TLQ40 30,31 was performed in non-disease human brain tissues (Supplementary Table S1). The specificity of the isoform-specific antibodies in immunofluorescence staining was confirmed using a p53-null cell line H358 with a $\Delta 133 p 53$, full-length $p 53$, or $p 53 \beta$ expression vector (Supplementary Figures S1A and B). As astrocytes were reported to be a major cell type showing p53 immunoreactivity in non-neoplastic human brain tissues, ${ }^{32,33}$ we investigated whether the p53 isoforms would also be expressed in astrocytes and performed co-staining with astrocyte-specific marker glial fibrillary acidic protein (GFAP) (Figure 1a and Supplementary Figure S1C). Both $p 53 \beta$ and $\Delta 133 p 53$ signals were detected in GFAP-positive cells and localized within 4',6-diamidino-2-phenylindole (DAPI)-positive nuclei (Figure 1a and Supplementary Figure S1C, arrows). Nuclei of GFAP-negative cells did not express the isoforms (Figure 1a, arrow heads). These findings indicate that astrocytes are the major cell type in the human brain that express $\Delta 133 p 53$ and $p 53 \beta$.

We examined whether in vitro cultured astrocytes also express the p53 isoforms. Primary human astrocytes ${ }^{34}$ were serially passaged with monitoring by $S A-\beta$-gal staining and p16 ${ }^{\text {INK4A }}$ (ref. 16) expression (Figures $1 \mathrm{~b}$ and $\mathrm{c}$ ) and those at early passage (passage number 5 ) and approaching senescence (passage number 13) were examined in western blot analysis (Figure 1c; Supplementary Figures S1D and E). Fulllength p53 was upregulated during senescence. The $\Delta 133 p 53-s p e c i f i c$ antibody detected a single band of $35 \mathrm{kDa}$ corresponding to $\Delta 133 p 53 a^{35}$ (simply called $\Delta 133 p 53$ in this manuscript) in early-passage astrocytes, which was diminished upon senescence. In contrast, p53 $\beta$ (47 kDa) was also upregulated upon senescence. These results indicate that human astrocytes express the p53 isoforms, $\Delta 133 p 53$ and $p 53 \beta$, whose expression profile at cellular senescence is conserved across different cell types. ${ }^{30,31}$

Autophagic degradation of $\Delta 133 p 53$ and SRSF3mediated regulation of $p 53 \beta$ in human astrocytes. To investigate whether increased $\mathrm{p} 53 \beta$ protein and decreased $\Delta 133 p 53$ protein in senescent astrocytes are due to regulation at the mRNA level, we performed qRT-PCR using RNA samples extracted from serially passaged astrocytes in vitro (passage numbers 5 and 13, as above). Similar levels of $\triangle 133 p 53 \mathrm{mRNA}$ were expressed at both passage numbers (Figure 2a). p53 $\beta$, by contrast, significantly increased at the mRNA level in astrocytes approaching senescence (Figure 2a). These data indicate that $\Delta 133 p 53$ expression is mainly regulated at the protein level, whereas $p 53 \beta$ is regulated at the mRNA level, as we previously reported in human fibroblasts and CD8 ${ }^{+}$T-lymphocytes. ${ }^{30,31}$

We tested whether $\Delta 133 p 53$ is degraded via selective autophagy in astrocytes, as we had previously reported in senescent fibroblasts and CD8 ${ }^{+}$T-lymphocytes. ${ }^{31,36}$ Senescent astrocytes (passage number 15) with low levels of $\Delta 133 p 53$ were treated with a pharmacological inhibitor of autophagy, bafilomycin $A 1,{ }^{37}$ whose action was confirmed by increased LC3-II ${ }^{38}$ (Figure 2b). Treatment with bafilomycin A1 resulted in the upregulation of $\Delta 133 p 53$ in senescent astrocytes (Figure 2b), suggesting that autophagic degradation of $\Delta 133 p 53$ contributes to its downregulation in these cells. The stabilization of $\Delta 133 p 53$ by bafilomycin $A 1$ was also observed in an immortalized human astrocyte cell line ${ }^{39,40}$ (Supplementary Figure S2A). Knockdown of p62/SQSTM1, which is a ubiquitin-binding adaptor specifically functioning in the selective autophagy pathway, ${ }^{41}$ also stabilized $\Delta 133 p 53$ (Figure 2c), further supporting the degradation of $\Delta 133 p 53$ via selective autophagy. In addition, whereas proteasome inhibitor MG132 stabilized full-length p53, $\Delta 133 p 53$ was not stabilized but rather decreased in astrocytes (Supplementary Figure S2B), as previously observed in fibroblasts, ${ }^{30}$ likely because of induction of autophagy by proteasome inhibition. ${ }^{42,43}$ As a splicing factor SRSF3 inhibits the 
a
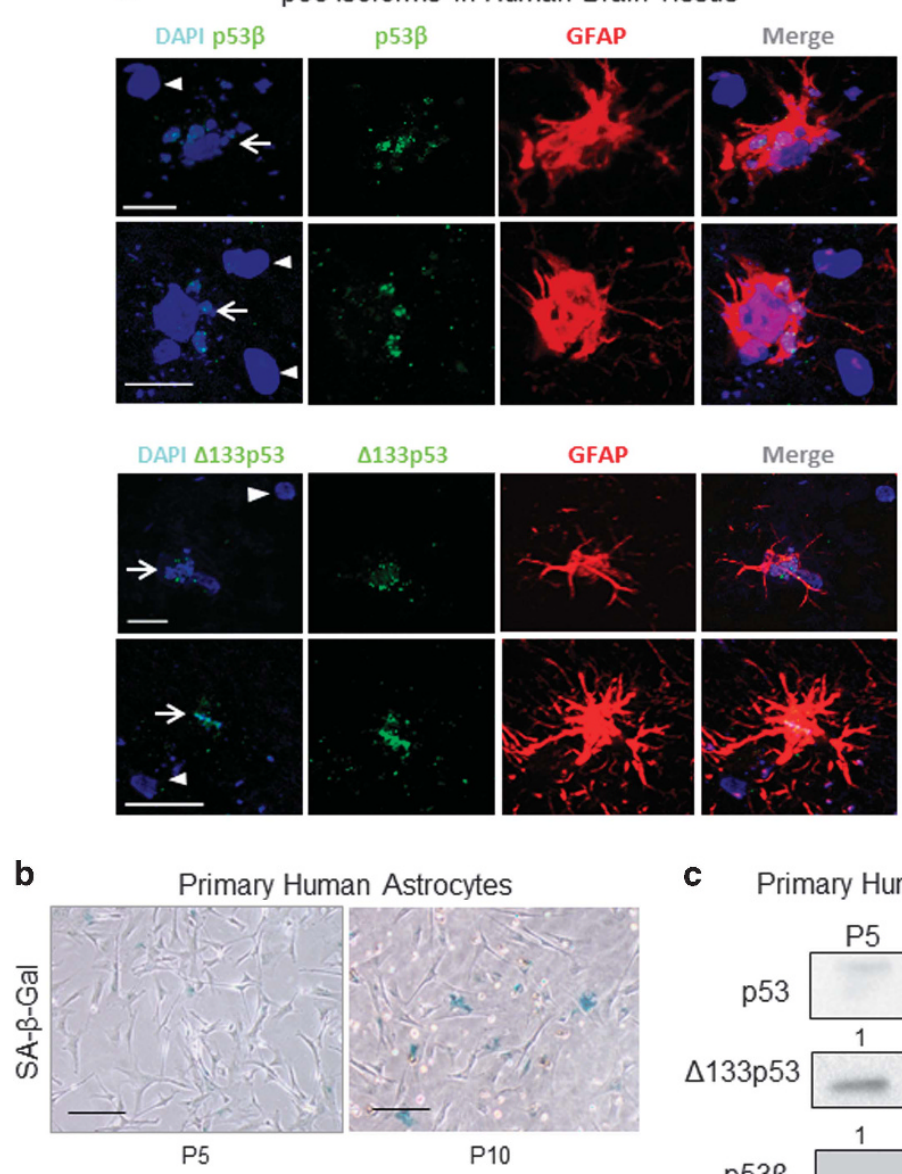

C Primary Human Astrocytes

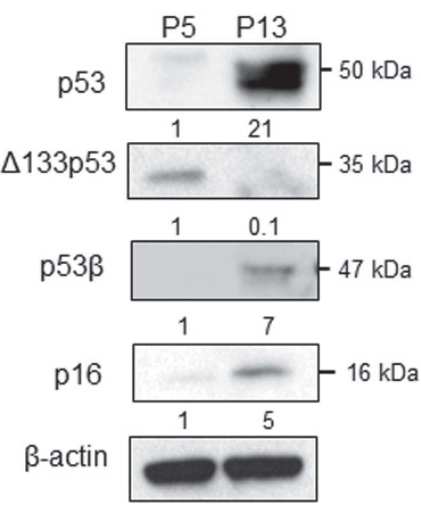

Figure 1 Astrocytes express $p 53$ isoforms, $\Delta 133 p 53$ and p53 $\beta$, and their expression is regulated during cellular senescence. (a) Immunofluorescence staining showing p53 $\beta$ and $\Delta 133 p 53$ expression in GFAP-positive astrocytes (arrows). No expression of p53 $\beta$ and $\Delta 133 p 53$ was observed in GFAP-negative cells (arrow heads). Top panel, nondisease (ND) tissue case 1; bottom panel, ND case 2. Scale bars $=10 \mu \mathrm{m}$. (b) SA- $\beta$-gal staining in primary human astrocytes at passage number 5 (P5) and P10. These astrocytes became completely growth-arrested at P15. Scale bars $=20 \mu \mathrm{m}$. (c) Western blot analysis of full-length p53, p53 isoforms, and senescence marker p1 $6^{\text {INK4A }}$ in earlypassage (P5) and aged (P13) primary human astrocytes. Densitometric values are normalized to $\beta$-actin

alternative mRNA splicing generating $\mathrm{p} 53 \beta$ in human fibroblasts and $\mathrm{CD}^{+}$T-lymphocytes, ${ }^{31,44}$ we examined whether SRSF3 regulates $p 53 \beta$ in astrocytes as well. Knockdown of SRSF3 through small interfering RNA (siRNA) in immortalized astrocytes resulted in a significant increase in p53 $\beta$ mRNA (Figure $2 d$ ), consistent with its negative regulation of $p 53 \beta$ at the transcript level. These results indicate that the regulatory mechanisms for p53 isoform expression (autophagic degradation of $\Delta 133 p 53$ and SRSF3-mediated regulation of $p 53 \beta$ splicing) are conserved in different cell types.

$\Delta 133 p 53$ knockdown or p53 $\beta$ overexpression induces cellular senescence and SASP in human astrocytes. We first recapitulated the senescence-associated expression signature (decreased $\Delta 133 p 53$ and increased $p 53 \beta$ ) in early-passage primary or immortalized human astrocytes to determine whether it induces these cells to senescence. Immortalized astrocytes were transfected with siRNA specifically targeting $\Delta 133 p 53^{45}$ or scramble siRNA control, followed by western blot analysis and immunofluorescence staining confirming $\Delta 133 p 53$ knockdown using $\Delta 133 p 53-$ specific antibody MAP4 $4^{30,31}$ (Figures $3 a$ and b; Supplementary Figure S3A). Three days following $\Delta 133 p 53$ knockdown, cells became growth-arrested (Figure 3c, top panel) with vacuolization (Figure $3 \mathrm{c}$, bottom panel) and increased SA- $\beta$-gal staining (Figures $3 d$ and $e$ ), which are characteristics of cellular senescence. Increased mRNA expression of pro-inflammatory cytokines IL- 6 and IL-1 $\beta$, as well as p21 ${ }^{\text {WAF1 }}$, was also observed in these senescent cells (Figure $3 f$ ), suggesting that loss of $\Delta 133 p 53$ induces SASP. 
a

Primary Human Astrocytes

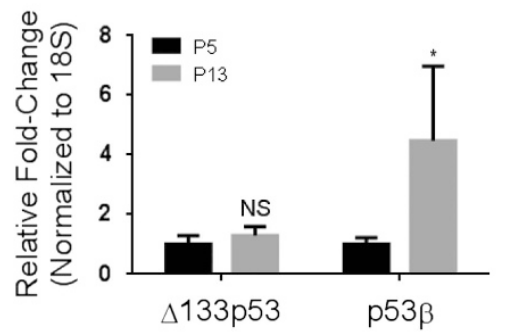

C

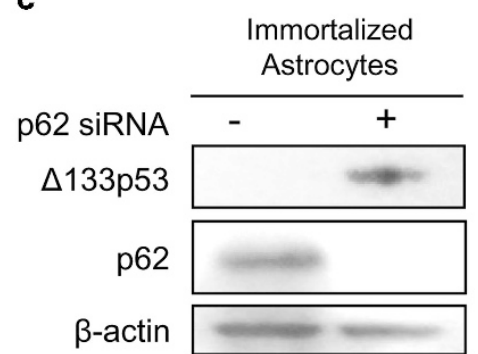

b

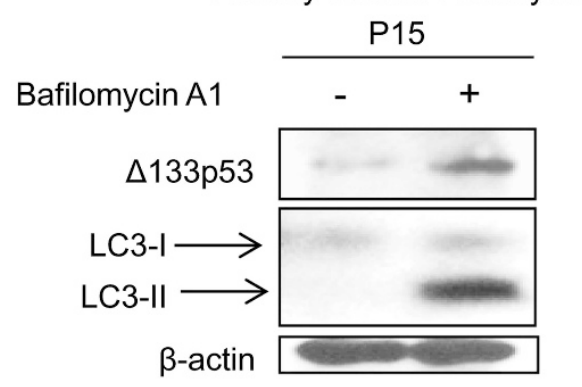

d Immortalized Astrocytes

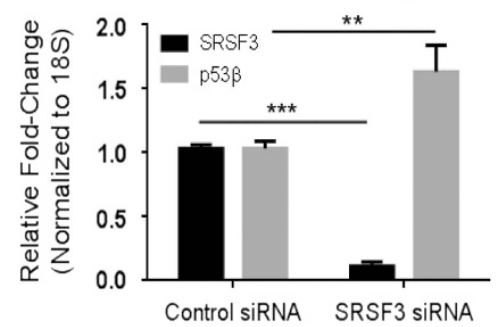

Figure 2 Autophagic degradation of $\Delta 133 p 53$ and SRSF3-mediated regulation of p53 $\beta$. (a) qRT-PCR analysis of $\Delta 133 p 53$ and p53 $\beta$ in early-passage (P5) and aged (P13) primary astrocytes. (b) $\Delta 133 p 53$ expression is restored by bafilomycin A1 treatment (100 nM for $4 \mathrm{~h}$ ) in senescent human astrocytes (P15). The activity of bafilomycin A1 was confirmed by increased LC3-II. (c) Knockdown of p62/SQSTM1 stabilizes $\Delta$ 133p53. Immortalized astrocytes were transfected with p62/SQSTM1 siRNA (+) or control siRNA (- ). (d) SRSF3 knockdown induces p53 $\beta$ mRNA. Immortalized astrocytes were transfected with SRSF3 siRNA or control siRNA and examined by qRT-PCR for SRSF3 and p53 $\beta$ expression. Immortalized astrocytes, which can be transfected with siRNA at a higher efficiency than primary astrocytes, were used in $\mathbf{c}$ and $\mathbf{d}$. Data are presented as mean \pm S.E.M. NS indicates $P>0.05,{ }^{\star} P \leq 0.05,{ }^{\star \star} P \leq 0.01,{ }^{\star \star \star} P \leq 0.001$ by unpaired two-tailed Student's $t$ test

Knockdown of $\Delta 133 p 53$ in early-passage primary astrocytes (confirmed by immunofluorescence; Supplementary Figure S3B) also resulted in increased SA- $\beta$-gal staining (Supplementary Figures S3C and D), the induction of SASP cytokines (Supplementary Figure S3E), and p21 WAF1 protein expression (Supplementary Figure S3F).

A lentiviral vector driving p53 $\beta$ expression or a vector control was transduced to immortalized astrocytes and its overexpression was confirmed by western blot analysis using a p53 $\beta$-specific antibody ${ }^{30,31}$ (Figure 4a; Supplementary Figure S4A). Similar to $\Delta 133 p 53$ knockdown, p53 $\beta$ overexpression inhibited cell proliferation (Figure $4 b$ ), increased SA- $\beta$-gal-positive cells (Figures $4 c$ and $d$ ), and induced proinflammatory SASP cytokines IL-6, IL-8, IL-1 $\beta$, and p21 WAF1 (Figure 4e). Early-passage primary astrocytes with p53 $\beta$ overexpression (Supplementary Figure S4B) were also growth-inhibited (Supplementary Figure S4C), had increased SA- $\beta$-gal staining (Supplementary Figure S4D-E), and induction of SASP (Supplementary Figure S4F). As p53 $\beta$ has been shown to promote apoptosis and senescence by differentially regulating gene expression in different cell types, ${ }^{29,46,47}$ cell viability as well as expression of Bax, p16 ${ }^{\mathrm{INK} 4}$, and p21 was also assessed (Supplementary Figures S4G-I). Percent cell viability was not significantly affected by overexpression of p53 $\beta$ (Supplementary Figure S4G). Consistently, there was no difference in expression of the apoptosis regulator Bax (Supplementary Figure S4H). In contrast, p21 and p16 expression were increased following $p 53 \beta$ overexpression (Supplementary Figures $\mathrm{S} 4 \mathrm{H}$ and I). These results indicate that $\Delta 133 p 53$ downregulation and $p 53 \beta$ upregulation have causative roles in inducing cellular senescence and SASP in astrocytes.

Increased neuronal death upon co-culture with $\Delta 133 p 53-$ knocked-down or p53 $\beta$-overexpressing astrocytes. To examine whether a phenotypic change in astrocytes (such as cellular senescence and SASP via $\Delta 133 p 53$ knockdown or $\mathrm{p} 53 \beta$ overexpression) alters astrocyte-to-neuron interaction, we utilized a co-culture system in which human primary astrocytes were co-plated with induced pluripotent stem cell (iPSC)-derived mature neurons, ${ }^{48}$ followed by assays for neuronal survival and death (Supplementary Figures S5A and B). Mature neurons were derived from iPSC (Supplementary Figure S5C) and confirmation of neuronal phenotypes was achieved through expression of sequential markers of differentiation: iPSCs expressed TRA-1-81 demonstrating pluripotency (Supplementary Figure S5D); neural stem cells (NSC) expressed Nestin and Sox1 (Supplementary Figure S5E); and neurons expressed neuronal nuclei marker (NeuN) (Supplementary Figure S5F). Motor neurons, a mature neuronal subtype that is primarily lost in ALS, were also derived from $\mathrm{iPSC}^{49}$ and their differentiation was confirmed by expression of non-phosphorylated neurofilament marker, SMI-32 (Supplementary Figure S5G).

When early-passage astrocytes with $\triangle 133 p 53$ siRNA or control siRNA (same cells as used in Supplementary Figure S3) were co-cultured with motor neurons for $48 \mathrm{~h}$, $\Delta 133 p 53-k n o c k e d-d o w n$, senescent astrocytes induced a higher number of neurons positive for cleaved caspase-3 
a
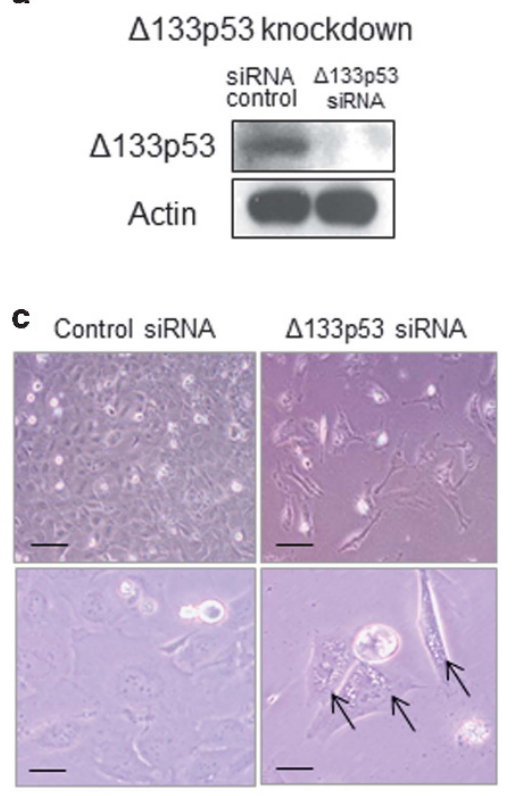

e Quantification of SA- $\beta$-gal

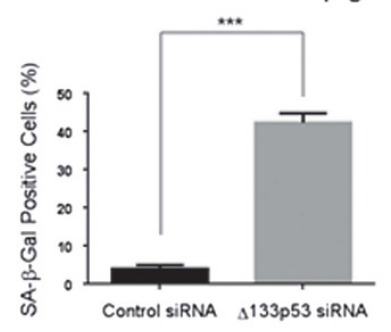

b
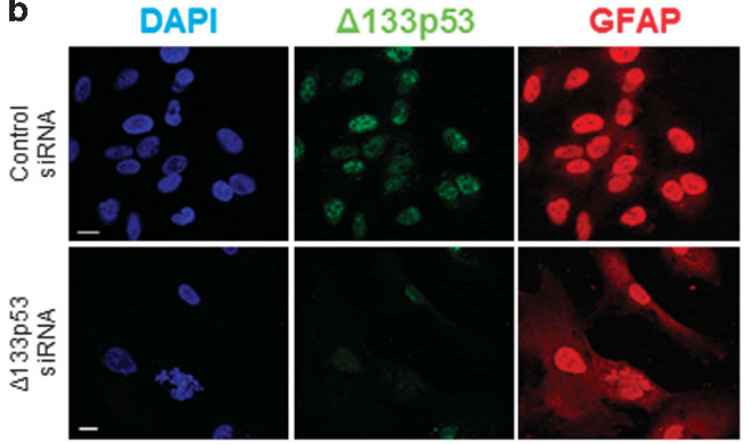

d

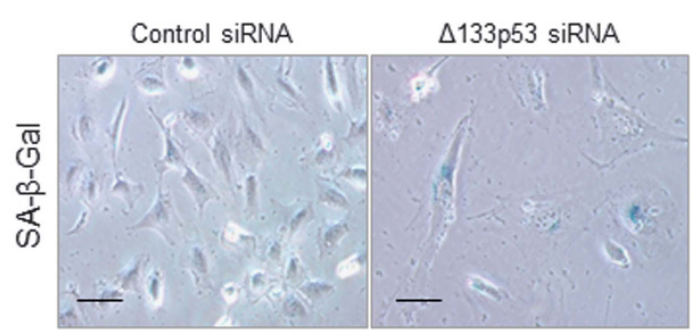

f

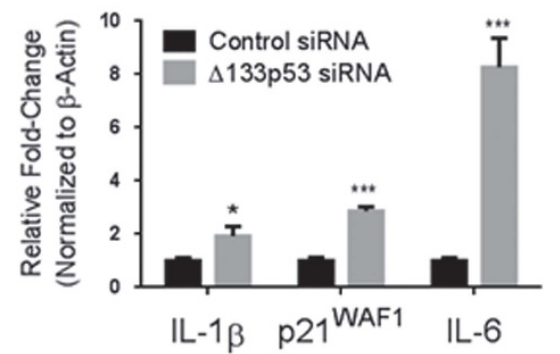

Figure 3 Knockdown of $\Delta 133 p 53$ induces senescent phenotype in astrocytes. Immortalized astrocytes were transfected with $\Delta 133 p 53$ siRNA or control siRNA and analyzed after 3 days. (a, b) Confirmation of $\Delta 133 p 53$ knockdown by western blot (a) and immunofluorescence (b). Scale bars $=10 \mu \mathrm{m}$. (c) Phase-contrast images of siRNA-transfected astrocytes (upper panel, $\times 20$ magnification; lower panel, $\times 40$ magnification). Arrows show vacuolization. Scale bars $=20 \mu \mathrm{m}$. (d, e) An increase in SA- $\beta$-gal staining by $\Delta 133$ p53 knockdown. Representative images, scale bars $=20 \mu \mathrm{m}$ (d) and quantitative summary from triplicated experiments (e). (f) Increase in IL-6, IL-1 $\beta$, and p21 WAF1 $\mathrm{mRNA}$ expression by $\Delta 133$ p53 knockdown. qRT-PCR was performed in triplicate. Data are presented as mean \pm S.E.M. ${ }^{*} P \leq 0.05,{ }^{* \star} P \leq 0.001$ by unpaired two-tailed Student's $t$ test

$(15.0 \pm 0.2 \%)$, a final effector of neuronal apoptosis, ${ }^{50}$ than control astrocytes $(1.3 \pm 0.3 \%)$ (Figures $5 a$ and b). Similarly, p53 $\beta$-overexpressing astrocytes (same cells as used in Supplementary Figure S4) also resulted in an increase in cleaved caspase-3-positive motor neurons (18.8 $\pm 1.0 \%)$ compared with vector control-transduced cells $(0.4 \pm 0.3 \%)$ (Figures $5 \mathrm{c}$ and $\mathrm{d}$ ). To examine the effects of astrocytes on neurons in general and to obtain another quantitative measure, the co-culture experiment was performed using less specialized neurons (generated as in Supplementary Figure S5C) and the number of surviving neurons (NeuNpositive) was counted after 48-h co-culture with astrocytes. A significant decrease in the number of surviving neurons was observed following co-culture with $\Delta 133 p 53-k n o c k e d-d o w n$ astrocytes (Figures $5 e$ and f) or p53 $\beta$-overexpressing astrocytes (Figures $5 \mathrm{~g}$ and $\mathrm{h}$ ). An increase in neuronal apoptosis by $p 53 \beta$-overexpressing astrocytes was also confirmed in this experimental setting (Supplementary Figures $\mathrm{S} 5 \mathrm{H}$ and I). These findings indicate that the senescence-associated p53 isoform expression signature confers astrocytes with neurotoxic activity.

$\Delta 133 p 53$ protects astrocytes from senescence and enhances their neuroprotective function. The induction of astrocyte senescence and neurotoxicity by reproducing the senescence-associated p53 isoform expression signature (Figures 3, 4, and 5) prompted us to hypothesize that manipulated expression of the p53 isoforms (which in this case recapitulates the expression profile in early-passage astrocytes) may also lead to senescence inhibition and neuroprotective function in astrocytes. To test this hypothesis, $\Delta 133 p 53$ expression was reconstituted via lentiviral vector transduction in primary astrocytes approaching senescence (passage number 12) (Figure 6a). Reconstituted expression of $\Delta 133 p 53$ restored cell proliferation (Figure $6 \mathrm{~b}$ ), decreased SA- $\beta$-gal-positive cells (Figures $6 b$ and $c$ ), and reduced the levels of pro-inflammatory SASP cytokines IL-6 and IL- $1 \beta$ (Figure 6d). Significantly, two neurotrophic growth factors, NGF and IGF-1, out of the three examined were two to 


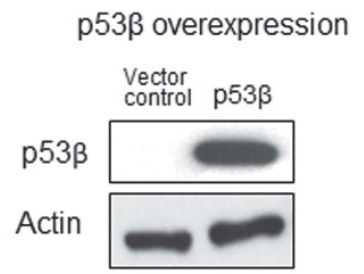

c Vector control

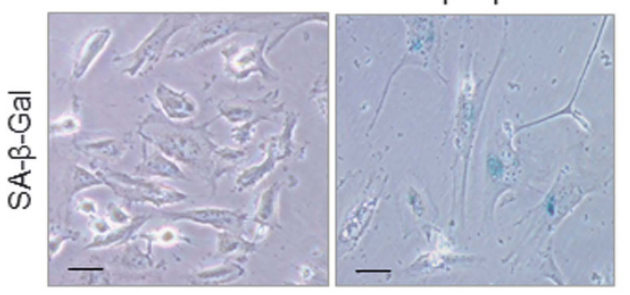

e

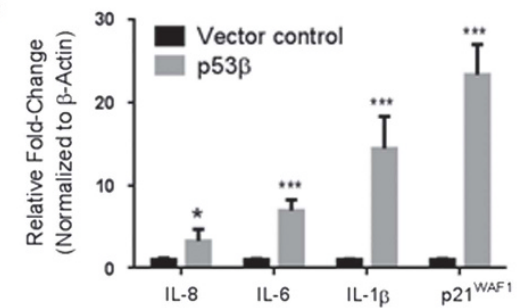

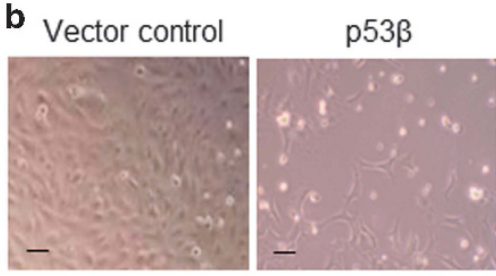

d

Quantification of SA- $\beta$-gal

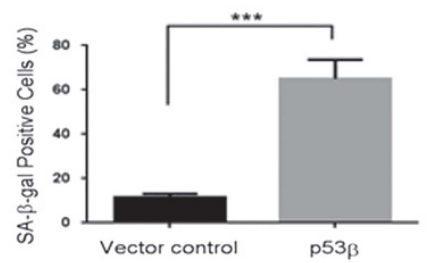

Figure 4 Overexpression of $p 53 \beta$ induces senescent phenotype in astrocytes. Immortalized astrocytes were transduced with p53 $\beta$ lentiviral vector or control vector and analyzed after 3 days. (a) Confirmation of p53 $\beta$ overexpression by western blot. (b) Phase-contrast images of transduced astrocytes. Scale bars $=20 \mu \mathrm{m}$. (c, d) An increase in $\mathrm{SA}-\beta$-gal staining by p53 $\beta$ overexpression. Representative images, scale bars $=20 \mu \mathrm{m}$ (c) and quantitative summary from triplicated experiments (d). (e) Elevated mRNA expression of IL-8, IL-6, IL-1 $\beta$, and p21 ${ }^{\text {WAF } 1}$ by p53 $\beta$ overexpression. qRT-PCR was performed in triplicate. Data are presented as mean \pm S.E.M. ${ }^{*} P \leq 0.05$, ${ }^{* * *} P \leq 0.001$ by unpaired two-tailed Student's $t$ test

threefold upregulated by $\Delta 133 p 53$ reconstitution (Figure $6 \mathrm{~d}$ ). Immunofluorescence staining of NGF confirmed its increased expression in $\Delta 133 p 53-$ reconstituted astrocytes (Supplementary Figure S6A), which was comparable with that in early-passage astrocytes (Supplementary Figure S6B).

We then used these $\Delta 133 p 53$-reconstituted astrocytes in the co-culture experiment (as performed in Figure 5). Vector control-transduced astrocytes (derived from passage number 12) resulted in a much larger number of motor neurons positive for cleaved caspase-3 $(35.0 \pm 2.0 \%$; Figures $6 e$ and f) than those derived from passage number $5(1.3 \pm 0.3 \%$; Figures $5 a-d)$, indicating a senescence-associated progression of neurotoxicity in astrocytes. The reconstitution of $\Delta 133 p 53$ in these near-senescent astrocytes significantly reduced the number of cleaved caspase-3-positive motor neurons ( 12\%; Figures $6 e$ and $f)$, suggesting that $\Delta 133 p 53$ in astrocytes functions to suppress neuronal apoptosis. Counting of NeuN-positive neurons following co-culture (Figures $6 \mathrm{~g}$ and $h$ ) also showed that aged astrocytes were less neuroprotective than early-passage astrocytes (compare controls in Figure $6 \mathrm{~h}$ versus Figures $5 f$ and $h$ ), and that reconstituted expression of $\Delta 133 p 53$ restored the number of surviving neurons back to the level exerted by early-passage astrocytes $(\Delta 133 p 53$ in Figure 6h; compare with controls in Figures $5 f$ and $h$ ).

To confirm the roles of NGF and IL-6 in $\Delta 133 p 53$-mediated astrocyte neuroprotective and neurotoxic function, neutralizing antibodies were used in co-culture experiments. As a positive control for IL-6-neutralizing antibody, we first performed immunofluorescence staining of untreated neurons and neurons treated with IL-6 alone or both IL-6 and IL-6neutralizing antibody (IL-6-NAb). Cleaved caspase-3 immunofluorescence staining was increased upon IL-6 treatment and reduced back to the control level upon co-incubation with IL-6-NAb (Supplementary Figure S6C). Consistently, the number of NeuN-positive surviving neurons was significantly reduced upon IL-6 treatment and restored by IL-6-NAb (Supplementary Figures S6C and D). These results confirmed that IL-6 promotes neurotoxicity and that IL-6-NAb is effective at neutralizing its function. Next, we used IL-6-NAb in co-culture experiments with neurons and aged astrocytes (P12) (Figure 6i, Supplementary Figure S6E). In these co-culture experiments, incubation with IL-6-NAb reduced neuronal apoptosis (indicated by cleaved caspase-3-positive neurons) to the level of $\Delta 133 p 53$-reconstituted astrocytes, but there was no combinatorial effect of $\triangle 133 p 53$ and IL-6-NAb over either alone (Figure 6i, Supplementary Figure S6E). In co-culture experiments with NGF-neutralizing antibody (NGF-NAb), the reduction in neuronal apoptosis by $\Delta 133 p 53$ reconstitution was abolished by NGF-NAb, and thus the level of neuronal apoptosis in $\Delta 133 p 53$-reconstited astrocytes with NGF-NAb was similar to that in control astrocytes with or without NGF-NAb (Figure 6i, Supplementary Figure S6E). These results indicate that $\Delta 133 p 53$ in astrocytes promotes 
a Early-passage astrocytes (P5) + motor neurons
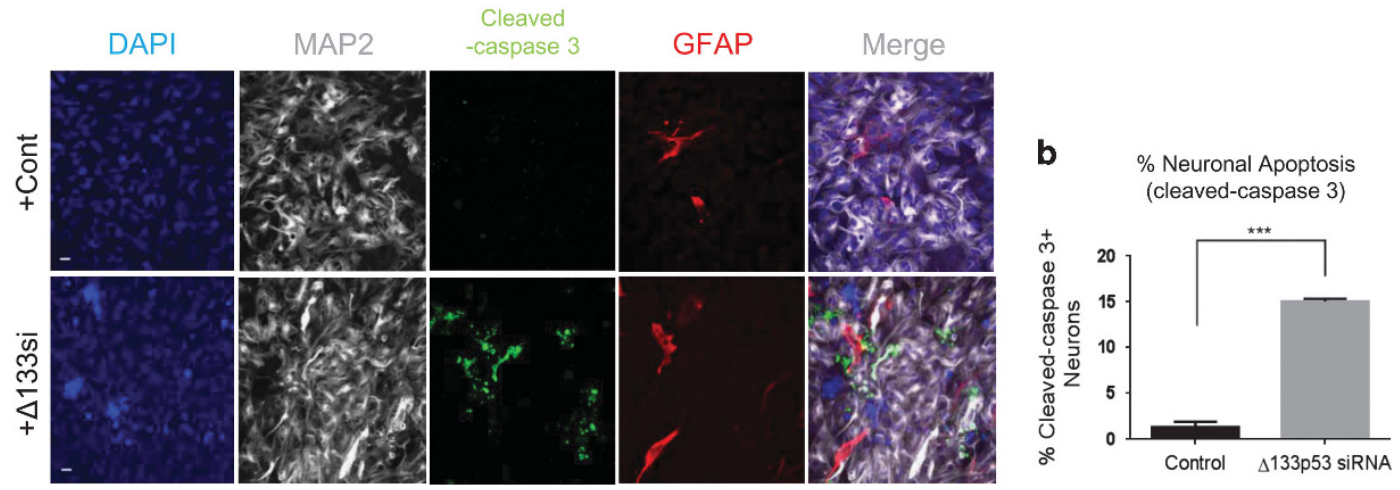

c

Early-passage astrocytes (P5) + motor neurons
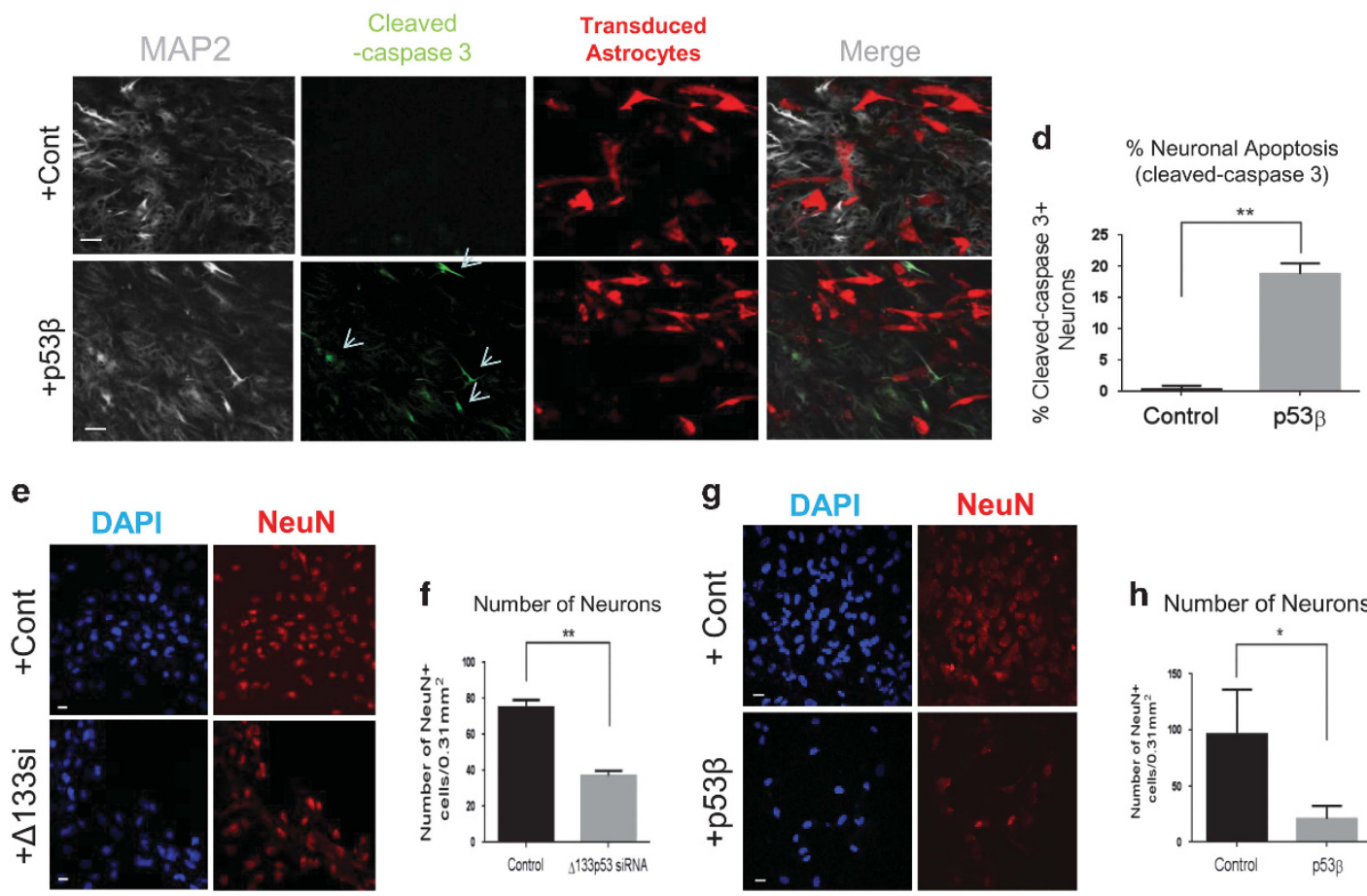

h Number of Neurons

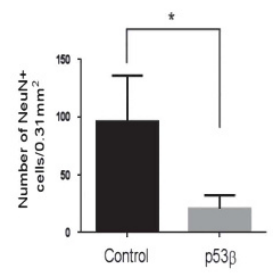

Figure 5 Increased neuronal death upon co-culture with $\Delta 133 p 53$-knocked-down or p53 $\beta$-overexpressing astrocytes. Early-passage primary astrocytes (P5) with $\Delta 133 p 53$ siRNA and control siRNA (generated as in Supplementary Figure S3) (a, b, e, and f) and those with p53 $\beta$-overexpression or control vector (generated as in Supplementary Figure S4) (c, d, g, and h) were used in co-culture for $48 \mathrm{~h}$ with motor neurons ${ }^{49}$ or less specialized neurons (as generated as in Supplementary Figures S5C-F). (a and $\mathbf{c}$ ) Immunofluorescence staining of MAP2 (neuronal marker) and cleaved caspase-3 (apoptosis marker). Astrocytes are marked by GFAP (a) or fluorescent signals derived from the lentiviral vectors (c). Scale bars $=10 \mu \mathrm{m}$. (b and $\mathbf{d})$ Quantification of neuronal apoptosis. Cleaved caspase-3-positive neurons per total number of MAP2-positive neurons were counted in triplicate experiments in five microscopic fields ( $\times 40$ magnification). (e and $\mathbf{g})$ Immunofluorescence staining of NeuN (neuronal marker). Scale bars $=10 \mu \mathrm{m}$. ( $f$ and $\mathbf{h}$ ) Quantification of number of neurons. Total number of NeuN-positive neurons were counted in at least five microscopic fields ( $\times 40$ magnification) in triplicate experiments. Data are presented as mean \pm S.E.M. ${ }^{*} P \leq 0.05,{ }^{* *} P \leq 0.01,{ }^{* \star *} P \leq 0.001$ by unpaired two-tailed Student's $t$ test

neuroprotection through upregulation of NGF and downregulation of IL-6.

Increased senescent astrocytes in brain tissues from neurodegenerative disease patients. To examine whether neurodegenerative disease tissues have increased features of cellular senescence, AD, ALS, age-matched non-disease, and non-disease pediatric tissues (Supplementary Table S1) were obtained. Immunohistochemical staining was performed using antibodies to proteins known to be associated with cellular senescence, such as $\mathrm{p} 16^{\mathrm{INK} 4 \mathrm{~A}},{ }^{16} \mathrm{NOS} 2,{ }^{20}$ and $\gamma \mathrm{H}_{2} \mathrm{AX}^{21}$ (Figure 7a). An increase in the senescenceassociated biomarkers was prominent in all neurodegenerative samples examined compared with controls (Figure 7a). Quantification of the number of $\mathrm{p} 16^{\mathrm{INK} 4 \mathrm{~A}}$-expressing cells revealed a significant increase in this senescence-associated gene in $A D$ and ALS tissues (Figure $7 b$ ). Although both $A D$ and ALS are associated with increased cellular senescence, qRT-PCR using brain tissue RNA samples showed that SASP cytokine IL-6 and a p53-inducible senescence 
a Vector control

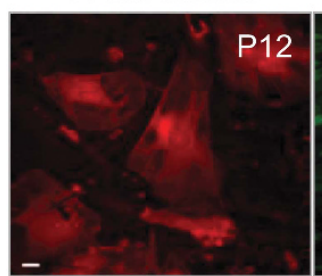

pLOC-RFP
$\Delta 133 p 53$

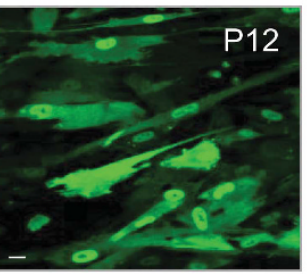

$\triangle 133 p 53-G F P$ b

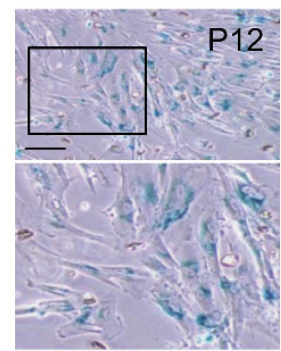

$\Delta 133 p 53$

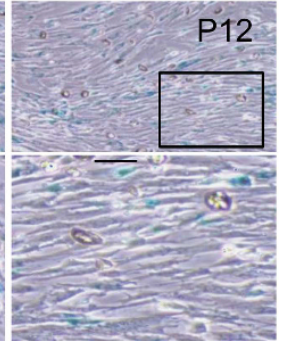

c

Quantification of SA- $\beta$-gal

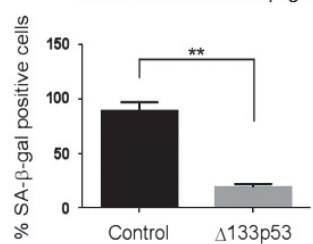

d

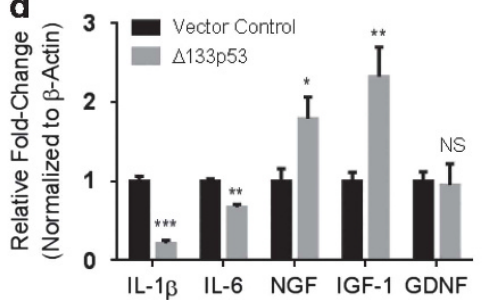

e

Aged astrocytes (P12) + motor neurons

Cleaved-caspase Transduced

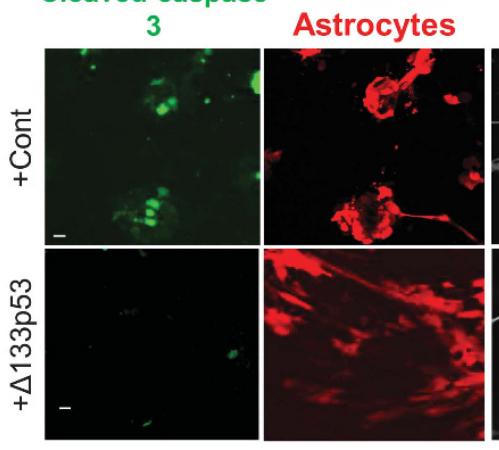

MAP2
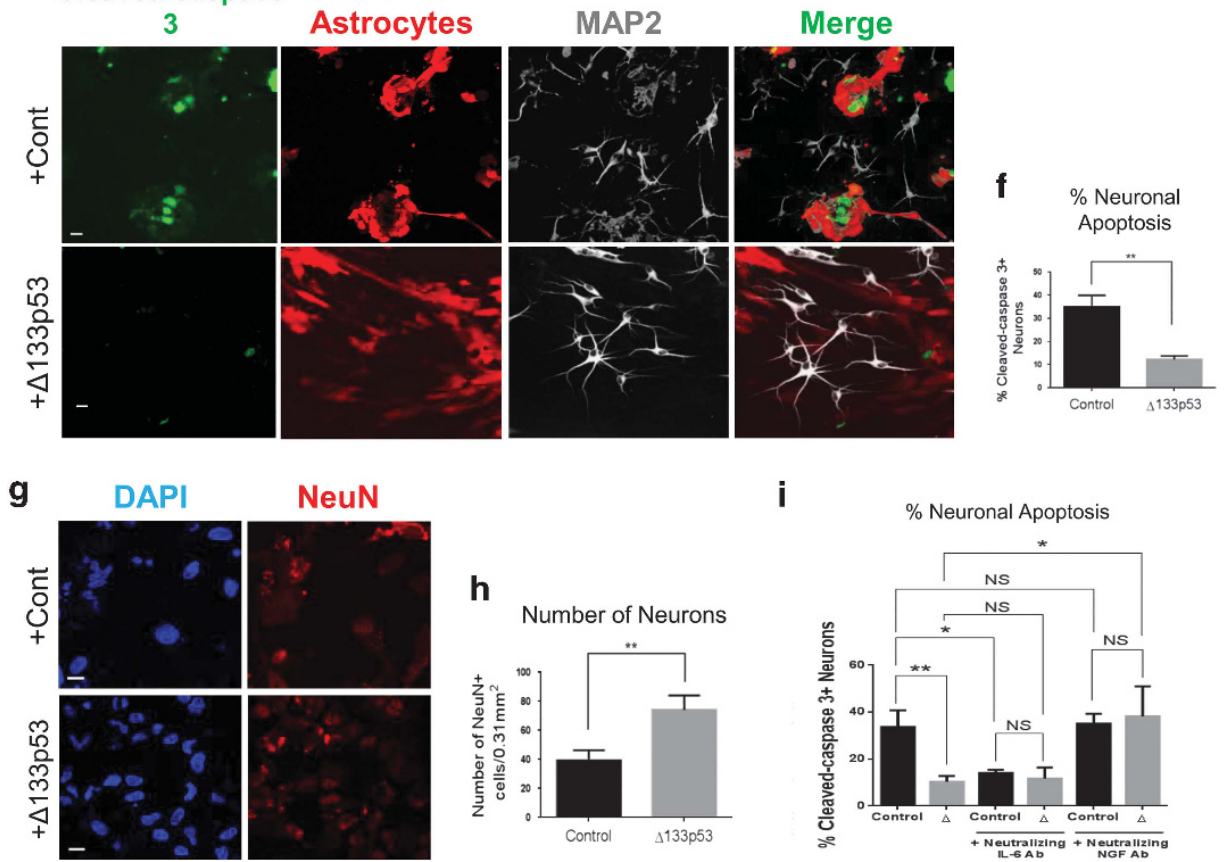

h

Number of Neurons

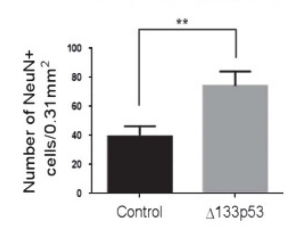

i

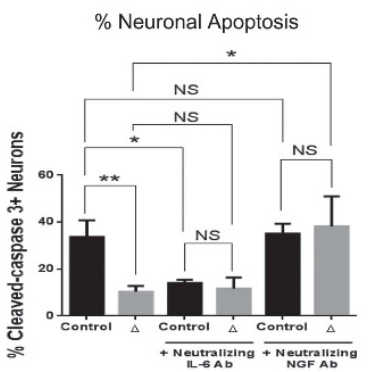

Figure $6 \Delta 133 p 53$ protects astrocytes from senescence and enhances neuroprotective function. Aged primary astrocytes (P12) were transduced with a lentiviral vector driving $\Delta 133$ p53 or its control vector and analyzed after 3 days. (a) Confirmation of lentiviral transduction by RFP (vector control) or GFP $(\Delta 133 p 53)$. Scale bars $=10 \mu \mathrm{m}$. (b) Decreased SA- $\beta$-gal staining by reconstituted expression of $\Delta 133$ p53. Representative images of SA- $\beta$-gal staining (top panels, scale bars $=20 \mu \mathrm{m}$ ) and enlarged images of the insets (bottom panels). (c) Quantitative summary of SA- $\beta$-gal staining from triplicated experiments. (d) Decreased expression of IL-1 $\beta$ and IL-6 and increased expression of NGF and IGF-1. qRT-PCR analysis was performed in triplicate. (e-h) $\Delta 133$ p53-reconstituted astrocytes and control astrocytes were used in co-culture with motor neurons (e and f) or less specialized neurons ( $\mathbf{g}$ and $\mathbf{~ h}$ ), as performed in Figure 5. (e) Decrease in cleaved caspse-3-positive motor neurons upon co-culture with $\Delta 133 p 53-$ reconstituted aged (P12) astrocytes. Immunofluorescence staining of cleaved caspase-3 and MAP2 was performed. Astrocytes were detected by the vector-derived fluorescence. Scale bars $=10 \mu \mathrm{m}$. (f) Quantification of neuronal apoptosis. Data were achieved by counting the number of cleaved caspase-3-positive neurons per total number of MAP2-positive neurons in triplicate experiments in five microscopic fields ( $\times 40$ magnification). (g) Immunofluorescence staining of NeuN. Scale bars $=10 \mu \mathrm{m}$. (h) Quantification of number of NeuN-positive neurons. Total number of NeuN-positive neurons were counted in at least five microscopic fields ( $\times 40$ magnification) in triplicate experiments. (i) Quantification of neuronal apoptosis in co-culture experiments with IL-6 or NGF-neutralizing antibodies. Data were from the experiment shown in Supplementary Figure S6E and achieved by counting the number of cleaved caspase-3-positive neurons per total number of NeuN-positive neurons in triplicate experiments in five microscopic fields ( $\times 40$ magnification). Data are presented as mean \pm S.E.M. NS indicates $P>0.05,{ }^{\star} P \leq 0.05,{ }^{\star \star} P \leq 0.01,{ }^{\star \star \star} P \leq 0.001$ by unpaired two-tailed Student's $t$ test 
a

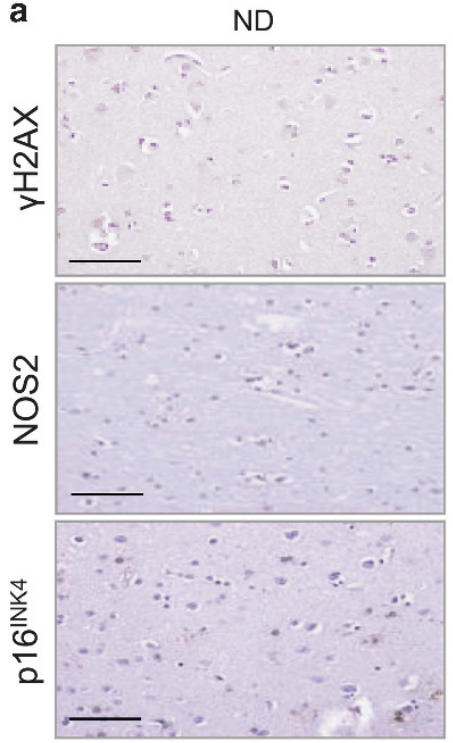

b

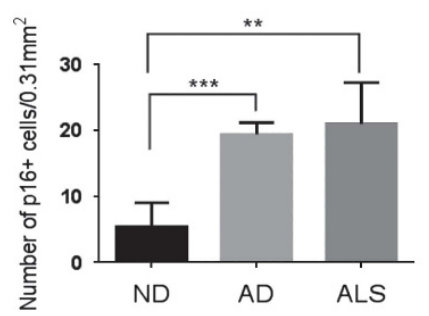

AD

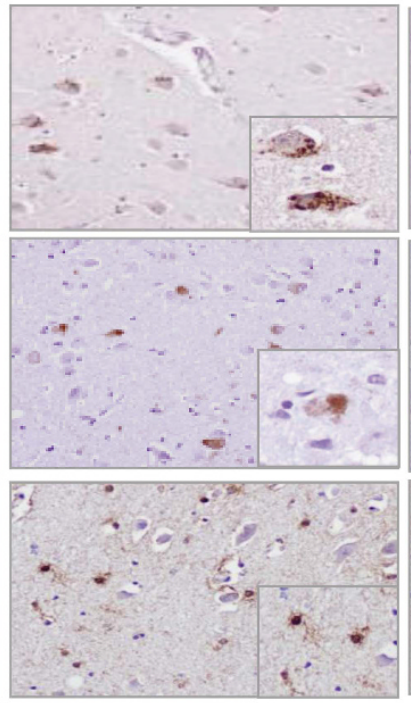

C Expression of senescence-associated genes

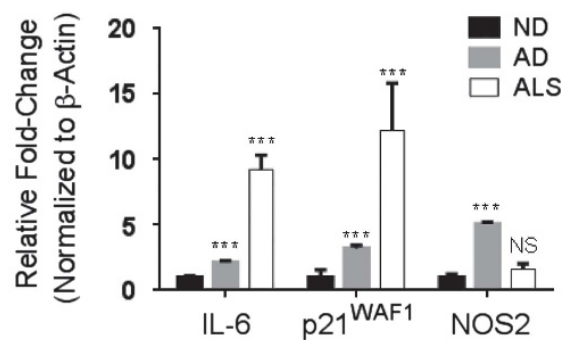

d

DAPI

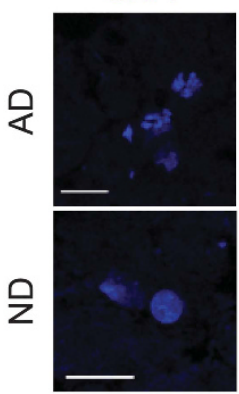

p16

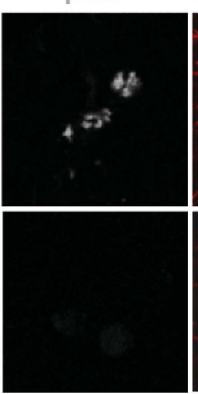

GFAP

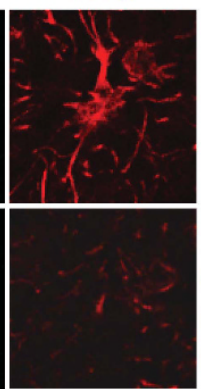

Merge

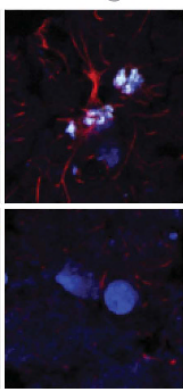

Figure 7 Increased senescent astrocytes in brain tissues from neurodegenerative disease patients. (a) Increased expression of senescence-associated proteins, $\gamma \mathrm{H} 2 \mathrm{AX}$, NOS2, and p16INK4A, in AD (case 5), and ALS (case 4). Non-disease (ND) age-matched control tissue was case 5. Scale bars $=50 \mu \mathrm{m}$. Insets in AD and ALS show enlarged positive cells. (b) Quantification of p16INK4A-positive cells in AD (cases 1-4) and ALS (cases 1-3) compared with ND (cases 1-4). (c) Elevated mRNA expression of senescence-associated genes in AD (cases 1-4) and ALS (cases 1-3) compared to ND (cases 1-4). qRT-PCR experiments were performed in triplicate. Data are presented as mean \pm S.E.M. (d) Increase in p16INK4A- and GFAP-positive cells in AD (case 1) compared to ND (case 1). Scale bars $=10 \mu \mathrm{m}$. NS indicates $P>0.05,{ }^{* \star} P \leq 0.01$, ${ }^{* * *} P \leq 0.001$ by unpaired two-tailed Student's $t$ test

regulator $\mathrm{p} 21^{\mathrm{WAF} 1}$ were upregulated more remarkably in ALS, while NOS2 upregulation was more evident in AD (Figure 7c), possibly reflecting the different disease pathologies. IL-6 and p2 $1^{\text {WAF1 }}$ expression levels were much lower in pediatric brain tissues versus aged brain tissues (Supplementary Figure S7A), indicating that these senescent changes not only are associated with neurodegenerative diseases, but also may occur during physiological brain aging. In agreement with astrocyte-like morphology of the senescent cells
(Figure 7a), immunofluorescence co-staining of $\mathrm{p} 16^{\mathrm{INK} 4 \mathrm{~A}}$ and GFAP as an astrocyte marker showed that the senescent cells were astrocytes (Figure 7d). Furthermore, both immunohistochemical (Supplementary Figure S7B) and immunofluorescence staining (Supplementary Figure S7C) of GFAP showed the presence of astrocytes with enlarged and flattened cytoplasms, which is characteristic of senescent cells, ${ }^{51}$ in $A D$ and ALS brain tissues, but not in non-disease control tissues. 
a

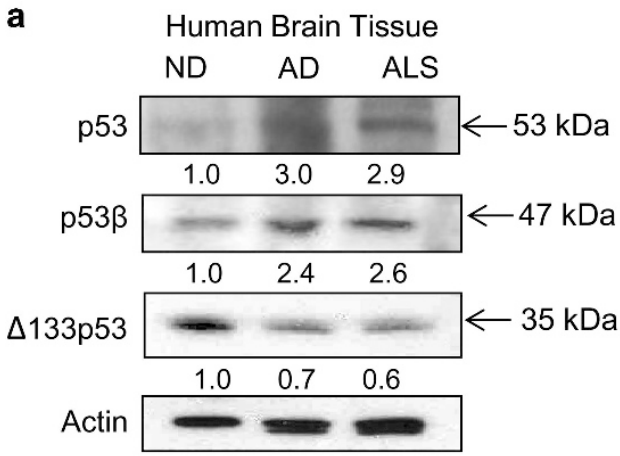

C

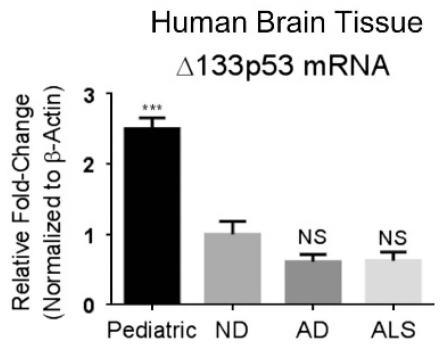

b

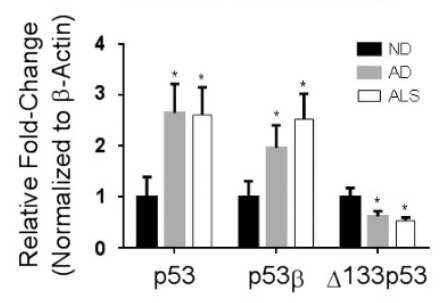

d

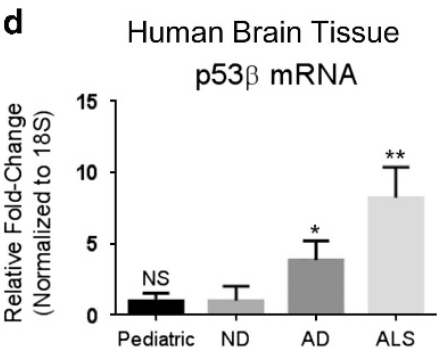

Figure $8 \quad p 53 \beta$ is upregulated and $\Delta 133 p 53$ is downregulated in neurodegenerative diseases. (a) Representative western blot showing elevated expression of full-length p53 and p53 $\beta$ and decreased $\Delta 133$ p53 expression in AD (case 3) and ALS (case 3) compared with non-disease (ND) age-matched control brain tissue (case 2). Densitometric values are normalized to $\beta$-actin. (b) Summary of densitometric analyses of western blots from AD (cases 1-4), ALS (cases 1-3), and ND (cases 1-4). (c and d) qRT-PCR analysis of $\Delta 133 p 53$ (c) and p53 $\beta$ (d) in pediatric brain tissue (cases 1-4), ND control (cases 1-4), AD (cases 1-4), and ALS (cases 1-3). Expression levels are shown relative to ND. Densitometric values are normalized to $\beta$-actin. Data are presented as mean \pm S.E.M. ${ }^{*} P \leq 0.05,{ }^{* *} P \leq 0.01,{ }^{* \star *} P \leq 0.001$ by unpaired two-tailed Student's $t$ test

p53 $\beta$ is upregulated and $\Delta 133 p 53$ is downregulated in neurodegenerative disease brains. To examine the expression of $p 53 \beta$ and $\triangle 133 p 53$ in $A D, A L S$, and agematched non-disease brain tissues, western blot analysis was performed using the $\triangle 133 p 53$-specific antibody MAP4 and the p53 $\beta$-specific antibody TLQ40, ${ }^{30,31}$ along with detection of fulllength p53. Only the $35 \mathrm{kDa} \Delta 133 \mathrm{p} 53$ bands corresponding to $\triangle 133 p 53 a$, but not smaller-size bands corresponding to $\Delta 133 p 53 \beta$ and $\Delta 133 p 53 \gamma$, were detected by MAP4 in these brain tissues (Supplementary Figure S8A). The major bands detected by TLQ40 were $47 \mathrm{kDa}$ in size, which corresponds to p53 $\beta$ that starts at the same methionine as full-length p53, with smaller amounts of $\mathrm{N}$-terminally truncated $\beta$ isoforms (Supplementary Figure S8B). These detection patterns are similar to those observed in primary human astrocytes (Figure 1c). We found that full-length p53 (53 kDa) and p53 $\beta$ (47 kDa) were upregulated, whereas $\Delta 133 p 53(35 \mathrm{kDa})$ was downregulated, in AD and ALS tissues compared with nondisease tissues (Figure 8a). Quantitative densitometric analysis determined that the upregulation of full-length p53 and p53 $\beta$ was two to threefold and the downregulation of $\Delta 133 p 53$ was 0.5-0.6-fold (Figure 8b). The upregulation of p53 $\beta$ and the downregulation of $\Delta 133 p 53$ in neurodegenerative diseases are consistent with the expression profiles of these p53 isoforms observed in senescent astrocytes in vitro (Figure 1c), as well as other in vivo conditions associated with increased senescence, such as pre-malignant colon adenomas ${ }^{30}$ and CD8 ${ }^{+}$T-lymphocytes in the elderly. ${ }^{31}$ The expression levels of $\triangle 133 p 53$ mRNA were not significantly changed between non-disease and AD or ALS tissues (Figure $8 \mathrm{c}$ ), while p53 $\beta$ mRNA was increased in $A D$ and ALS compared with non-disease tissues (Figure $8 d$ ), again in agreement with the observations in senescent astrocytes in vitro (Figure $2 \mathrm{a}$ ) and $\mathrm{CD} 8^{+}$T-lymphocytes in vivo. ${ }^{31} \mathrm{~A}$ higher level of expression of $\Delta 133 p 53$ mRNA in non-disease pediatric tissues compared with non-disease aged brain tissues (Figure 8c) may also suggest a transcriptional control of $\Delta 133 p 53$ during physiological brain development and aging. Thus, AD and ALS are associated with increased astrocyte senescence and the senescence-associated p53 isoform expression signature, validating that our in vitro experiments using serially passaged human astrocytes recapitulate part of in vivo pathology of these neurodegenerative diseases.

\section{Discussion}

Astrocytes exert both neuroprotective and neurodegenerative effects in a context-dependent manner, which are associated with either repression or induction of SASP, respectively. ${ }^{52}$ The data are consistent with the notion that the astrocyte-mediated effects of the p53 isoforms on neurons are exerted through their regulatory roles for SASP (Figures $3 f, 4 e$, and $6 \mathrm{~d}$ and i, Supplementary Figure S6E). One main feature of astrocyte SASP driving neuronal loss is the release of pro-inflammatory cytokines such as IL-6. ${ }^{53}$ Consistent with the neurodegenerative role of these SASP cytokines, the induction of SASP in astrocytes either by $\Delta 133 p 53$ knockdown (Figure $3 f$ ) or by p53 $\beta$ overexpression (Figure $4 \mathrm{e}$ ) leads to increased neuronal apoptosis (Figures $5 \mathrm{a}-\mathrm{d}$ ), while the repression of astrocyte SASP by $\Delta 133 p 53$ restoration (Figure $6 \mathrm{~d}$ ) leads to increased neuronal survival (Figures 6 e and f). Our data suggest that IL-6 is a therapeutic target in astrocytes to prevent neurotoxicity (Figure 9b). 


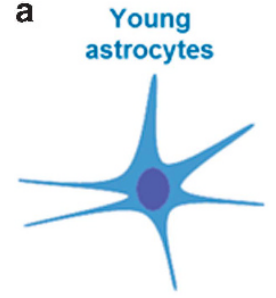

Neuroprotective growth factors (NGF)

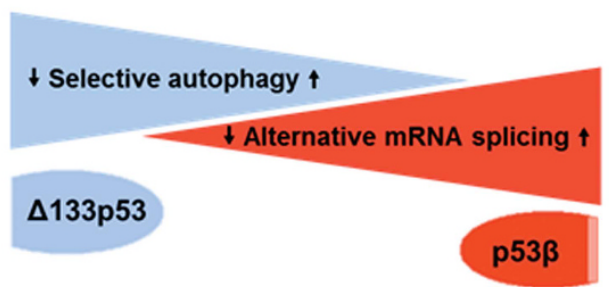

$\mathrm{p} 53 \beta$

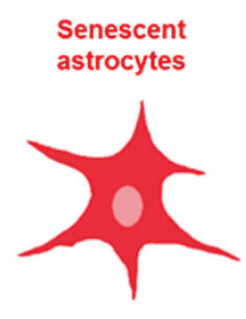

Neurotoxic SASP cytokines (IL-6)
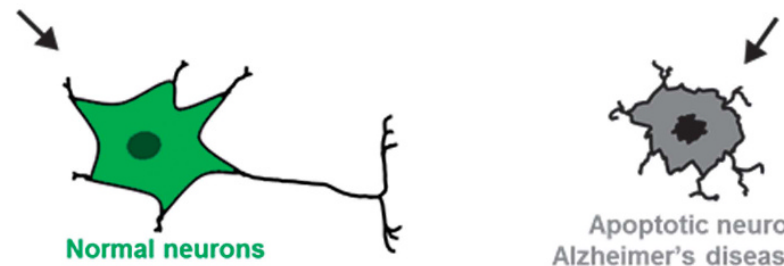

Apoptotic neurons:

Alzheimer's disease, ALS

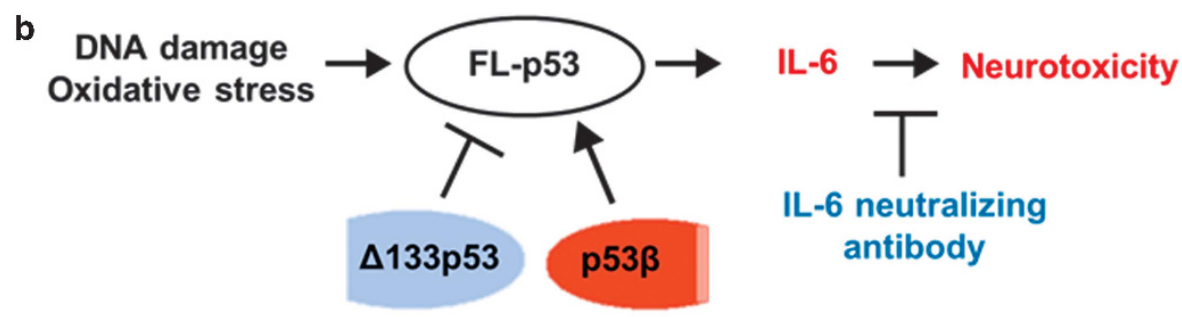

Figure 9 Proposed models of the p53 isoform regulation of astrocyte-mediated neuroprotection and neurodegeneration. (a) The p53 isoforms regulate astrocyte-mediated neuroprotection and neurodegeneration. $\Delta 133 \mathrm{p} 53$, which is regulated by selective autophagy-mediated degradation, ${ }^{36}$ is abundant in early-passage astrocytes in vitro and astrocytes in non-disease brain tissues (here collectively termed 'young') and contributes to the neuroprotective function of these 'young' astrocytes. p53 $\beta$, which is generated via alternative mRNA splicing, ${ }^{44}$ is induced in senescent astrocytes in vitro and in neurodegenerative disease brain tissues and contributes to the neurotoxic effect of these senescent astrocytes. (b) IL-6 is a therapeutic target in astrocytes to prevent neurotoxicity. DNA damage and oxidative stress activate full-length p53 (FL-p53), which leads to transcriptional activation of IL-6 ${ }^{57}$ and neurotoxicity. The modulation of FL-p53 activity by the p53 isoforms, as well as IL-6-neutralizing antibody, prevents neurotoxicity

The data also indicate that the upregulation of neurotrophic growth factors such as NGF and IGF-1 in astrocytes (Figure 6d, Supplementary Figure S6A) mediates $\Delta 133 p 53-$ induced neuroprotection (Figures $6 \mathrm{~g}-\mathrm{i}$, Supplementary Figure S6E). NGF and IGF-1 are known to promote neuronal survival ${ }^{54,55}$ and are decreased in Alzheimer's and Parkinson's disease brain tissues. ${ }^{11,12}$ Furthermore, astrocytes utilize IGF-1 to protect neurons from oxidative stress. ${ }^{56}$ Although this study focused on astrocyte-to-neuron signaling, possible roles of the p53 isoforms in neurons and neuron-toastrocyte signaling in neurodegenerative disease deserve further investigation.

Full-length p53 is known to transactivate pro-inflammatory cytokine genes such as IL-6 and IL- $8 .{ }^{57}$ It is thus likely that the effect of p53 $\beta$ or $\Delta 133 p 53$ on SASP and neurodegeneration is at least in part through cooperation with or dominant-negative inhibition of full-length p53. ${ }^{30,31}$ As full-length p53 functions to inhibit the IGF-1 signaling pathway, ${ }^{58}$ the dominant-negative inhibition by $\Delta 133 p 53$ of full-length $p 53$ activity ${ }^{30}$ may increase IGF-1 signaling towards neuroprotection. Another possibility is that $\Delta 133 p 53$ may directly upregulate the neurotrophic factors NGF and IGF-1 through its gain-of-function activity, which was recently reported to activate a set of genes for DNA damage repair. ${ }^{59}$ Although in silico analysis of the NGF and IGF-1 gene promoters did not identify a perfect match to the predicted $\Delta 133 p 53$-binding response element, ${ }^{59}$ further studies will elucidate the regulation of these neurotrophic factors by $\Delta 133 p 53$ in dominant-negative and gain-of-function manners.

A phenotypic shift from neurotoxic SASP astrocytes to neuroprotective astrocytes may represent a promising therapeutic approach for inhibiting or delaying the progression of neurodegenerative diseases. In the proposed model (Figure 9 ), enhancement of $\Delta 133 p 53$ activity and/or inhibition of $\mathrm{p} 53 \beta$ activity could lead to such phenotypic shift of astrocytes. Because increased neurotoxicity, which was exerted by in vitro aged astrocytes and reverted by reconstituted expression of $\Delta 133 p 53$ in this study, is also characteristic of astrocytes derived from patients with neurodegenerative diseases, ${ }^{60,61}$ our findings have implications in developing therapeutic interventions. This study provides a rationale for exploration of small molecules that can modulate the expression level or activity of the p53 isoforms to inhibit the progression of neurodegeneration. Finally, only humans and other primates have the equivalent of $\Delta 133 p 53$ (Supplementary Figure S9). It is interesting whether this primate-specificity of $\Delta 133 p 53$ is related to primate-specific accumulation of $\beta$-amyloid ${ }^{62}$ and other physiological or pathological processes specific or preferential to humans and other primates. 


\section{Materials and Methods}

Primary cells and cell lines. Primary human astrocytes were obtained from Sciencell (Carlsbad, CA, USA). ${ }^{34}$ They were maintained in Astrocyte Medium supplemented with $2 \%$ fetal bovine serum, $1 \%$ astrocyte growth supplement from Sciencell, and $1 \%$ penicillin/streptomycin solution. When confluent, cells were split at a ratio of 1:4 (earlier passages) to 1:2 (later passages) until they reached replicative senescence. For immortalized human astrocytes, human fetal brain material was dissected from 8- to 12-week-old abortuses, aspirated through a 19gauge needle, washed twice with Eagle's minimum essential medium and plated onto poly-D-lysine $(0.1 \mathrm{mg} / \mathrm{ml})$-coated flasks. After 3 weeks, cells were transfected using calcium phosphate precipitation with a plasmid DNA containing the SV40 mutant with a deletion of its origin of replication (pMK16). ${ }^{39,40} \mathrm{H} 358$ cells were purchased from American Type Culture Collection (Manassas, VA, USA).

Plasmid constructs. To generate retroviral expression vectors of human p53 isoforms, Flag-tagged p53 $\beta$ was PCR-amplified using pSVp53 $\beta$ and pSVDNp53, ${ }^{29}$ respectively, as templates and then inserted into Notl and EcoRI sites of a pQCXIN vector (BD Biosciences, Franklin Lakes, NJ, USA). These constructs were verified by DNA sequencing. The Precision LentiORF RFP control vector (which drives RFP as an ORF insert, as well as IRES-translated GFP from the pLOC lentiviral vector backbone) was purchased from Open Biosystems/GE Dharmacon (Lafayette, CO, USA). For overexpression of $p 53 \beta$ and $\Delta 133 p 53$, the RFP insert was replaced with cDNA inserts of $p 53 \beta$ and $\Delta 133 p 53$, respectively.

Retroviral and lentiviral transduction. The retroviral constructs were transfected into Phoenix packaging cells (Orbigen, Inc., San Diego, CA, USA) using Lipofectamine 2000 (Invitrogen, Paisley, Renfrewshire, UK). Lentiviral supernatants were obtained using the Trans-lentiviral packaging system (Open Biosystems). Retro- and lentiviral vector supernatants were collected $48 \mathrm{~h}$ after transfection and used to infect cells in the presence of polybrene $(8 \mu \mathrm{g} / \mathrm{ml}$; Sigma-Aldrich, St. Louis, MO, USA). Two days after infection, the cells were selected with G418 (600 $\mu \mathrm{g} / \mathrm{ml}$; Sigma-Aldrich), puromycin $(2 \mu \mathrm{g} / \mathrm{ml}$; Sigma-Aldrich) or zeocin ( $1 \mathrm{mg} /$ $\mathrm{ml}$; Invitrogen)

Transfection of siRNA oligonucleotides. siRNA oligonucleotides were transfected at a final concentration of $12 \mathrm{nM}$ using Lipofectamine RNAiMAX (Invitrogen). The following oligonucleotides were from Invitrogen Stealth Select siRNA targeting $\triangle 133 p 53: 5^{\prime}$-GGAGGUGCUUACACAUGUU-3', ${ }^{45}$ p62/SQSTM1: $5^{\prime}$-AGAAGUGGACCCGUCUACAGGUGAA- $3^{\prime},{ }^{36}$ SRSF3: 5'-AGAGCUAGAUGGAA GAACATT-3, ${ }^{44}$ and Stealth non-specific RNAi negative control (no. 12,935-100).

SA- $\beta$-gal staining and cell treatments. SA- $\beta$-gal staining was performed with the Senescence- $\beta$-Galactosidase Staining Kit (Cell Signaling Technology, Danvers, MA, USA). Bafilomycin A1 was obtained from Sigma-Aldrich and incubated for $4 \mathrm{~h}$ at a concentration of $100 \mathrm{nM}$. MG132 was obtained from LC Laboratories (Woburn, MA, USA) and incubated for $8 \mathrm{~h}$ at a concentration of $15 \mu \mathrm{M}$.

Cell viability assay. Cell pellets were resuspended in $20 \mathrm{ul}$ of Trypan blue and cell viability was calculated using a TC20 Automated Cell Counter (Bio-Rad, Hercules, CA, USA).

Neuronal differentiation of iPSCs. The iPSC line, i20 (NIH stem cell bank), was differentiated to NSCs, and then to neurons using Gibco Pluripotent Stem Cell Neural Induction (Life Technologies, Frederick, MD, USA). For motor neuron differentiation, iPSCs were grown to $80 \%$ confluency, then digested with collagenase IV (Invitrogen) for $8 \mathrm{~min}$. Cells were scraped off of the dish, and after settling, the supernatant was aspirated, and cells were re-plated into low adherence dishes (Corning, Corning, NY, USA) in KSR (Invitrogen)-based media with $20 \mathrm{ng} / \mathrm{ml}$ FGF (R\&D Systems, Minneapolis, MN, USA), $20 \mu \mathrm{M}$ ROCK-I (Tocris, Bristol, UK), $10 \mu \mathrm{M}$ SB431542 (Tocris), and $0.2 \mu \mathrm{M}$ LDN193189 (Stemgent). Embryoid bodies were transitioned to a KSR-free medium after 3 days. Retinoic acid was added to the media after 5 days to direct the cells towards a rostral spinal cord phenotype, with additional patterning using $1 \mu \mathrm{M}$ smoothened agonist (Calbiochem, Billerica, MA, USA) and $0.5 \mu \mathrm{M}$ purmorphamine (Stemgent, Cambridge, MA, USA) after 7 days to ventralize the differentiating population. After 14-16 days in suspension, the embryoid bodies were dissociated and plated on dishes coated with polyornithine or poly-D-lysine and laminin for an additional 7-14 days. Neuronal cultures were maintained in neurobasal media (Invitrogen) with $25 \mu \mathrm{M}$ glutamate (Sigma, St. Louis, MO, USA), $0.4 \mu \mathrm{g} / \mathrm{ml}$ ascorbic acid (Sigma), $10 \mathrm{ng} / \mathrm{ml}$ GDNF
(Sigma), $10 \mathrm{ng} / \mathrm{ml}$ CNTF (Sigma), $1 \mu \mathrm{g} / \mathrm{ml}$ laminin (BD Biosciences). B-27, N2, non-essential amino acids, and pen/strep/glutamine were all from Invitrogen. Two days after plating, $10 \mathrm{nM}$ dihydrotestosterone was added, and the cultures were maintained for an additional 7-14 days.

Co-culture system. Following differentiation of iPSC to NSCs, NSCs were plated at a density of $3 \times 10^{4} \mathrm{cells} / \mathrm{cm}^{2}$ in a four-well chamber slide coated with $20 \mu \mathrm{g} / \mathrm{ml}$ poly-L-ornithine and $10 \mu \mathrm{g} / \mathrm{ml}$ laminin. Media was changed to neural differentiation media (1 × Neurobasal Medium, 2\% B-27 Serum-Free Supplement, 2 mM GlutaMAX-1 Supplement, Life Technologies) after 2 days. Media was changed every 3 days and NSCs were allowed to mature neurons for 1 month. Primary human astrocytes were plated on top of the mature neurons at a density of $3 \times 10^{4} \mathrm{cells} / \mathrm{cm}^{2}$. Media was changed to a $1: 1$ ratio of astrocyte (Sciencell) and neuron differentiation medium. ${ }^{48,63,64}$ After $48 \mathrm{~h}$, cells were fixed with $4 \%$ paraformaldehyde for immunocytochemistry staining. Percent apoptosis was calculated by counting number of cleaved caspase-3-, MAP2-, or NeuN-positive neurons in triplicate experiments in five microscopic fields ( $\times 40$ magnification) for three biological replicates. In addition, the number of neurons remaining after the $48 \mathrm{~h}$ co-culture period was also quantified with five frames per condition for three biological replicates and counting the number of NeuN-positive neurons.

Recombinant IL-6 and neutralizing antibody treatments. Recombinant IL-6 (Invivogen, San Diego, CA, USA) was incubated for $24 \mathrm{~h}$ at a concentration of $5 \mathrm{ng} / \mathrm{ml}$. In experiments with neutralizing antibodies, co-culture was performed as above with NGF neutralizing antibody (Alomone labs, Jerusalem, Israel) at a concentration of $500 \mathrm{ng} / \mathrm{ml}$ or IL-6 neutralizing antibody (Invivogen) at a concentration of $5 \mu \mathrm{g} / \mathrm{ml}$. After $48 \mathrm{~h}$, cells were fixed and stained as described above.

Immunohistochemistry and immunofluorescence. Frozen and formalin-fixed paraffin embedded human tissue sections were washed in PBS before blocking for $1 \mathrm{~h}$ in PBS containing $0.1 \%$ Triton-X and $10 \%$ donkey serum (Sigma-Aldrich). Donkey serum is used to block non-specific binding sites before incubation with primary antibody overnight at $4^{\circ} \mathrm{C}$. Antigens were detected using the antibodies listed in Supplementary Table S2. After overnight incubation, they were washed in PBS three times for $10 \mathrm{~min}$, before incubation with the appropriate conjugated secondary antibodies for $1 \mathrm{~h}$ at room temperature. The secondary antibody was conjugated to fluorophores: Alexa-488, -568, and -647 (Invitrogen; 1:400). After washing in PBS three times for $10 \mathrm{~min}$, sections were incubated for $10 \mathrm{~min}$ in 4',6-diamidino-2-phenylindole (10ug/ml, Sigma-Aldrich) to counterstain the cell nuclei, and rinsed three times for $10 \mathrm{~min}$ in $0.1 \mathrm{M}$ phosphate buffer. Sections were mounted and slides coverslipped with FluorSave mounting medium (Millipore, Billerica, MA, USA). Omission of primary antibody was used as a negative control in all immunofluorescence experiments. For immunohistochemistry on paraffin sections, slides were heated to $65^{\circ} \mathrm{C}$ before immersion in histoclear and rehydration with graded alcohols. Sections were blocked in 1\% H2O2 in PBS-Tween 20 (PBS-T) and then in 5\% normal goat serum in PBS-T. Antigens were detected using the antibodies listed in Supplementary Table S2. Binding of the primary antibody was detected using a biotinylated secondary antibody listed in Supplementary Table S3 with an ABC standard kit (Vector Laboratories, Burlingame, CA, USA). Visualization was enabled using a $0.05 \%$ diaminobenzene hydrochloride solution (Sigma-Aldrich). Omission of primary antibody was used as negative controls in all immunohistochemistry experiments.

Immunocytochemistry. Cells were washed with PBS and fixed for $10 \mathrm{~min}$ with $4 \%$ paraformaldehyde. Cells were permeabilized with $0.01 \%$ Triton-X for $4 \mathrm{~min}$, washed with PBS and then blocked in 5\% FBS for $1 \mathrm{~h}$ at room temperature. Primary antibodies listed in Supplementary Table S2 were applied overnight at $4^{\circ} \mathrm{C}$. Cells were washed with PBS before incubation with a secondary antibody conjugated to fluorophores: Alexa-488, 568- and -647 at a dilution of 1:400 (Life Technologies) and 4',6-diamidino-2-phenylindole for $1 \mathrm{~h}$. Coverslips were mounted on to slides with FluorSave mounting medium (Chemicon). Omission of primary antibody was used as a negative control in all immunocytochemistry experiments.

Immunoblotting. Cells and tissues were lysed in RIPA buffer. Lysates were kept on ice for $30 \mathrm{~min}$ prior to sonication. Protein concentration was measured using the Bradford assay method. NuPAGE $4 \times$ loading buffer was added to all lysates and then boiled for $5 \mathrm{~min}$. Then, $40 \mu \mathrm{g}$ of protein was loaded onto a Tris-glycine gel 
(Thermo Scientific, Waltham, MA, USA) for electrophoresis. Proteins were then transferred onto a PVDF membrane. Membranes were blocked in 1:1 mixture of Superblock and Tris-Buffered Saline (TBS, $125 \mathrm{mM}$ Tris and $200 \mathrm{mM} \mathrm{NaCl}$ ), containing $0.1 \%$ Tween-20. Membranes were incubated in the primary antibodies listed in Supplementary Table S2 overnight at $4{ }^{\circ} \mathrm{C}$, and washed three times in TBSTween-20. Membranes were then incubated in secondary antibody listed in Supplementary Table S3 for $1 \mathrm{~h}$ at room temperature and the signal visualized SuperSignal developing reagent and visualized using X-ray films (Fujifilm, Tokyo, Japan).

RNA extraction and cDNA preparation. mRNA was extracted using the RNeasy Mini Kit (Qiagen, Crawley, UK) according to the manufacturer's instructions. For mouse brain tissue, $10-20 \mathrm{mg}$ of frozen cortical tissue was added to $300 \mu \mathrm{l}$ lysis buffer containing $0.001 \% \beta$-mercaptoethanol. Tissues or cells were homogenized and lysate mixed 1:1 with $70 \%$ ethanol and centrifuged through an RNeasy Mini Spin column. The column was washed and treated with DNAse 1 for $15 \mathrm{~min}$, before washing again to remove contaminants. RNA was eluted with RNase-free water. The abundance and quality of the resulting RNA was assessed using a Nanodrop ND-1000 spectrophotometer (Nanodrop Technologies, Wilmington, DE, USA). RNA samples were diluted so that $200 \mathrm{ng}$ total RNA could be used for a $25-\mu l$ reverse-transcription reaction. cDNA was synthesized using SuperScript II Reverse Transcriptase (Invitrogen).

Quantitative real-time polymerase chain reaction (qRT-PCR). For the quantitative analysis of mRNA expression, the Tecan Sunrise 7500 real-time PCR system (Applied Biosystems, Foster City, CA, USA) was used, with the DNA binding dye SYBR Green (Qiagen) or Taqman (Life Technologies) primers for the detection of PCR products. Each reaction was performed in triplicate using $2 \mu \mathrm{l}$ of CDNA in a final volume of $20 \mu \mathrm{l}$. The following thermal cycle was used for all samples: $10 \mathrm{~min}-95^{\circ} \mathrm{C}$; 40 cycles of $30 \mathrm{~s}-95^{\circ} \mathrm{C}, 40 \mathrm{~s}$-primer specific annealing temperatures, $40 \mathrm{~s}-72{ }^{\circ} \mathrm{C}$. The melting points, optimal conditions, and specificities of the reactions were first determined using a standard procedure. The expression level of each target gene was analyzed based on the $\Delta \Delta \mathrm{Ct}$ method and the results expressed as relative expression normalized to $18 \mathrm{~S}$ or $\beta$-actin. Primers for $\Delta 133 \mathrm{p} 53, \mathrm{p} 53 \beta$, and $18 \mathrm{~S}$ were purchased from Invitrogen and their sequences are as follows: $\triangle 133$ p53: forward 5'-TGACTTTCAACTCTGTCTCCTTCCT-3'; reverse 5'-GGCCAGACCATCGCTATCTG-3'. p53 $\beta$ : forward 5'-GCGAGCACTGCCCAACA-3'; reverse $5^{\prime}$-GAAAGCTGGTCTGGTCCTGA-3'. 18S: forward 5'-GTAACCCGTTGAA CCCCATT-3'; reverse 5'-CCATCCAATCGGTAGTAGCG-3.' Taqman primer assays for IL-6, p21, NOS2, IL-1- $\beta$, and $\beta$-actin were purchased from Life Technologies (sequences available from Life Technologies)

Statistical analysis. Data are presented as mean \pm standard error of the mean (S.E.M.) of an $n=3$ independent experiments unless otherwise stated. Statistical comparisons were made using unpaired two-tailed Student's $t$-test. Differences were considered significant at a value of ${ }^{*} P \leq 0.05,{ }^{* *} P \leq 0.01$, ${ }^{* \star *} P \leq 0.001$. ImageJ software was used to quantify gel bands from immunoblots using densitometry.

\section{Conflict of Interest}

The authors declare no conflict of interest.

Acknowledgements. We thank R Douglas Fields and Bríd Ryan for comments to the manuscript and Kenneth Fischbeck for providing reagents and materials. Confocal microscopy was supported by the National Cancer Institute. Human brain tissues were obtained from the Georgetown University Brain Bank and the Human Brain Collection Core, NIMH. This work was primarily funded by the National Cancer Institute, NIH. CT was supported by the National Institutes of Health-Oxford Scholars Program. BV is supported with projects P206/12/G151 and MEYS-NPS I-LO1413. $\mathrm{XL}$ is supported by the Ludwig Institute for Cancer Research Ltd. BTH is partially supported by Georgetown University Brain Bank, NIMH Brain Core, and Target ALS grant.

\section{Author contributions}

$\mathrm{CT}, \mathrm{IH}, \mathrm{BTH}$, and $\mathrm{CCH}$ conceived and designed the project. $\mathrm{CT}, \mathrm{IH}$, and EF performed the experiments. $\mathrm{CT}, \mathrm{IH}, \mathrm{BTH}$, and $\mathrm{CCH}$ analyzed data. EOM, BV, DPL, and $\mathrm{XL}$ provided reagents and guidance on the project. All authors wrote the manuscript.
1. Wimo A, Prince M. World Alzheimer Report 2010: The Global Economic Impact of Dementia. Alzheimer's Disease International: London, UK, 2010.

2. Larkindale J, Yang W, Hogan PF, Simon CJ, Zhang Y, Jain A et al. Cost of illness for neuromuscular diseases in the United States. Muscle Nerve 2014; 49: 431-438.

3. Barres BA. The mystery and magic of glia: a perspective on their roles in health and disease. Neuron 2008; 60: 430-440.

4. Verkhratsky A, Sofroniew MV, Messing A, deLanerolle NC, Rempe D, Rodríguez JJ et al. Neurological diseases as primary gliopathies: a reassessment of neurocentrism. ASN Neuro 2012; 4: 131-149.

5. Furman JL, Sama DM, Gant JC, Beckett TL, Murphy MP, Bachstetter AD et al. Targeting astrocytes ameliorates neurologic changes in a mouse model of Alzheimer's disease. J Neurosci 2012; 32: 16129-16140.

6. Das MM, Svendsen CN. Astrocytes show reduced support of motor neurons with aging that is accelerated in a rodent model of ALS. Neurobiol Aging 2014; 36: 1130-1139.

7. Haidet-Phillips AM, Hester ME, Miranda CJ, Meyer K, Braun L, Frakes A et al. Astrocytes from familial and sporadic ALS patients are toxic to motor neurons. Nat Biotechnol 2011; 29: 824-828.

8. Jia JP, Meng R, Sun YX, Sun WJ, Ji XM, Jia LF. Cerebrospinal fluid tau, Abeta1-42 and inflammatory cytokines in patients with Alzheimer's disease and vascular dementia. Neurosci Lett 2005; 383: 12-16.

9. Campbell IL, Abraham CR, Masliah E, Kemper P, Inglis JD, Oldstone MB et al. Neurologic disease induced in transgenic mice by cerebral overexpression of interleukin 6. Proc Natl Acad Sci USA 1993; 90: 10061-10065.

10. Farina C, Aloisi F, Meinl E. Astrocytes are active players in cerebral innate immunity. Trends Immunol 2007; 28: 138-145.

11. Nagatsu T, Mogi M, Ichinose $H$, Togari A. Changes in cytokines and neurotrophins in Parkinson's disease. J Neural Transm Suppl 2000: 277-290.

12. Tuszynski MH. Nerve growth factor gene therapy in Alzheimer disease. Alzheimer Dis Assoc Disord 2007; 21: 179-189.

13. Erickson Kl, Prakash RS, Voss MW, Chaddock L, Heo S, McLaren M et al. Brain-derived neurotrophic factor is associated with age-related decline in hippocampal volume. J Neurosci 2010; 30: 5368-5375.

14. Brenner M, Johnson AB, Boespflug-Tanguy $\mathrm{O}$, Rodriguez D, Goldman JE, Messing A. Mutations in GFAP, encoding glial fibrillary acidic protein, are associated with Alexander disease. Nat Genet 2001; 27: 117-120.

15. Miller RG, Mitchell JD, Lyon M, Moore DH. Riluzole for amyotrophic lateral sclerosis (ALS)/ motor neuron disease (MND). Cochrane Database Syst Rev 2007; CD001447.

16. Rayess $\mathrm{H}$, Wang MB, Srivatsan ES. Cellular senescence and tumor suppressor gene p16. Int J cancer 2012; 130: 1715-1725.

17. Roninson IB. Oncogenic functions of tumour suppressor p21Waf1/Cip1/Sdi1: association with cell senescence and tumour-promoting activities of stromal fibroblasts. Cancer Lett 2002; 179: 1-14.

18. Debacq-Chainiaux F, Erusalimsky JD, Campisi J, Toussaint O. Protocols to detect senescence-associated beta-galactosidase (SA-betagal) activity, a biomarker of senescent cells in culture and in vivo. Nat Protoc 2009; 4: 1798-1806.

19. Campisi J. Aging, cellular senescence, and cancer. Annu Rev Physiol 2013; 75 685-705.

20. Sohn JJ, Schetter AJ, Yfantis HG, Ridnour LA, Horikawa I, Khan MA et al. Macrophages, nitric oxide and microRNAs are associated with DNA damage response pathway and senescence in inflammatory bowel disease. PLOS ONE 2012; 7: e44156.

21. Wang C, Jurk D, Maddick M, Nelson G, Martin-Ruiz C, von Zglinicki T. DNA damage response and cellular senescence in tissues of aging mice. Aging Cell 2009; 8: 311-323.

22. Coppé J-P, Desprez P-Y, Krtolica A, Campisi J. The senescence-associated secretory phenotype: the dark side of tumor suppression. Annu Rev Pathol 2010; 5: 99-118.

23. Demaria M, Ohtani N, Youssef SA, Rodier F, Toussaint W, Mitchell JR et al. An essential role for senescent cells in optimal wound healing through secretion of PDGF-AA. Dev Cell 2014; 31: 722-733.

24. Serrano M. Senescence helps regeneration. Dev Cell 2014; 31: 671-672.

25. Banito A, Lowe SW. A new development in senescence. Cell 2013; 155: 977-978.

26. Storer M, Mas A, Robert-Moreno A, Pecoraro M, Ortells MC, Di Giacomo V et al. Senescence is a developmental mechanism that contributes to embryonic growth and patterning. Cell 2013; 155: 1119-1130.

27. Muñoz-Espín D, Cañamero M, Maraver A, Gómez-López G, Contreras J, Murillo-Cuesta S et al. Programmed cell senescence during mammalian embryonic development. Cell 2013; 155: 1104-1118.

28. Zilfou JT, Lowe SW. Tumor suppressive functions of p53. Cold Spring Harb Perspect Bio 2009; 1: a001883.

29. Bourdon J-C, Fernandes K, Murray-Zmijewski F, Liu G, Diot A, Xirodimas DP et al. p53 isoforms can regulate p53 transcriptional activity. Genes Dev 2005; 19: 2122-2137.

30. Fujita K, Mondal AM, Horikawa I, Nguyen GH, Kumamoto K, Sohn JJ et al. p53 isoforms Delta133p53 and p53beta are endogenous regulators of replicative cellular senescence. Nat Cell Biol 2009; 11: 1135-1142.

31. Mondal AM, Horikawa I, Pine SR, Fujita K, Morgan KM, Vera E et al. p53 isoforms regulate aging- and tumor-associated replicative senescence in T lymphocytes. J Clin Invest 2013; 123: 5247-5257.

32. Mendrysa SM, Ghassemifar S, Malek R. p53 in the CNS: perspectives on development, stem cells, and cancer. Genes Cancer 2011; 2: 431-442. 
33. Kurtkaya-Yapicier O, Scheithauer BW, Hebrink D, James CD. p53 in nonneoplastic central nervous system lesions: an immunohistochemical and genetic sequencing study. Neurosurgery 2002; 51: 1246-1254; discussion 1254-5.

34. Bitto A, Sell C, Crowe E, Lorenzini A, Malaguti M, Hrelia S et al. Stress-induced senescence in human and rodent astrocytes. Exp Cell Res 2010; 316: 2961-2968.

35. Surget S, Khoury MP, Bourdon J-C. Uncovering the role of p53 splice variants in human malignancy: a clinical perspective. Onco Targets Ther 2013; 7: 57-68.

36. Horikawa I, Fujita K, Jenkins LM, Hiyoshi Y, Mondal AM, Vojtesek B et al. Autophagic degradation of the inhibitory p53 isoform $\Delta 133 p 53 \alpha$ as a regulatory mechanism for p53-mediated senescence. Nat Commun 2014; 5: 4706

37. Mizushima N, Yoshimori T, Levine B. Methods in mammalian autophagy research. Cell 2010; 140: 313-326.

38. Tanida I, Ueno T, Kominami E. LC3 and sutophagy. Methods Mol Biol 2008; 445: 77-88.

39. Ferenczy MW, Johnson KR, Steinberg SM, Marshall LJ, Monaco MC, Beschloss AM et al. Clonal immortalized human glial cell lines support varying levels of JC virus infection due to differences in cellular gene expression. J Neuroimmune Pharmacol 2013; 8 : 1303-1319.

40. Major EO, Miller AE, Mourrain P, Traub RG, de Widt E, Sever J. Establishment of a line of human fetal glial cells that supports JC virus multiplication. Proc Natl Acad Sci USA 1985; 82 1257-1261.

41. Johansen $T$, Lamark T. Selective autophagy mediated by autophagic adapter proteins Autophagy 2011; 7: 279-296.

42. Wu WK, Wu YC, Yu L, Li ZJ, Sung JJY, Cho CH. Induction of autophagy by proteasome inhibitor is associated with proliferative arrest in colon cancer cells. Biochem Biophys Res Commun 2008; 374: 258-263.

43. Pandey UB, Nie Z, Batlevi Y, McCray BA, Ritson GP, Nedelsky NB et al. HDAC6 rescues neurodegeneration and provides an essential link between autophagy and the UPS. Nature 2007; 447: 859-863.

44. Tang Y, Horikawa I, Ajiro M, Robles Al, Fujita K, Mondal AM et al. Downregulation of splicing factor SRSF3 induces p53 $\beta$, an alternatively spliced isoform of p53 that promotes cellular senescence. Oncogene 2013; 32: 2792-2798.

45. Bernard H, Garmy-Susini B, Ainaoui N, Van Den Berghe L, Peurichard A, Javerzat S et al. The p53 isoform, $\Delta 133 p 53 \alpha$, stimulates angiogenesis and tumour progression. Oncogene 2013; 32: 2150-2160

46. Marcel V, Fernandes K, Terrier O, Lane DP, Bourdon J-C. Modulation of $p 53 \beta$ and $p 53 \gamma$ expression by regulating the alternative splicing of TP53 gene modifies cellular response. Cell Death Differ 2014; 21: 1377-1387.

47. Marcel V, Dichtel-Danjoy M-L, Sagne C, Hafsi H, Ma D, Ortiz-Cuaran S et al. Biological functions of p53 isoforms through evolution: lessons from animal and cellular models. Cell Death Differ 2011; 18: 1815-1824.
48. Haidet-phillips AM, Hester ME, Miranda CJ, Meyer K, Braun L, Frakes A et al. Astrocytes from familial and sporadic ALS patients are toxic to motor neurons. Nat Biotechnol 2012; 29: 824-828.

49. Grunseich C, Zukosky K, Kats IR, Ghosh L, Harmison GG, Bott LC et al. Stem cell-derived motor neurons from spinal and bulbar muscular atrophy patients. Neurobiol Dis 2014; 70: 12-20.

50. Zhao M, Su J, Head E, Cotman CW. Accumulation of caspase cleaved amyloid precursor protein represents an early neurodegenerative event in aging and in Alzheimer's disease. Neurobiol Dis 2003; 14: 391-403.

51. Kuilman T, Michaloglou C, Mooi WJ, Peeper DS. The essence of senescence. Genes Dev 2010; 24: 2463-2479.

52. Pertusa M, García-Matas S, Rodríguez-Farré E, Sanfeliu C, Cristòfol R. Astrocytes aged in vitro show a decreased neuroprotective capacity. J Neurochem 2007; 101: 794-805.

53. Jiang T, Cadenas E. Astrocytic metabolic and inflammatory changes as a function of age. Aging Cell 2014; 13: 1059-1067.

54. Hefti F. Nerve growth factor promotes survival of septal cholinergic neurons after fimbrial transections. J Neurosci 1986; 6: 2155-2162.

55. Wine RN, McPherson CA, Harry GJ. IGF-1 and pAKT signaling promote hippocampal CA1 neuronal survival following injury to dentate granule cells. Neurotox Res 2009; 16: 280-292.

56. Genis L, Dávila D, Fernandez S, Pozo-Rodrigálvarez A, Martínez-Murillo R, Torres-Aleman I. Astrocytes require insulin-like growth factor I to protect neurons against oxidative injury. F1000Res 2014; 3: 28

57. Lowe JM, Menendez D, Bushel PR, Shatz M, Kirk EL, Troester MA et al. p53 and NF-kB coregulate proinflammatory gene responses in human macrophages. Cancer Res 2014; 74: 2182-2192.

58. Levine AJ, Feng Z, Mak TW, You H, Jin S. Coordination and communication between the p53 and IGF-1-AKT-TOR signal transduction pathways. Genes Dev 2006; 20: 267-275.

59. Gong L, Gong H, Pan X, Chang C, Ou Z, Ye S et al. p53 isoform $\Delta 113 p 53 / \Delta 133 p 53$ promotes DNA double-strand break repair to protect cell from death and senescence in response to DNA damage. Cell Res 2015; 25: 351-369.

60. Ilieva $\mathrm{H}$, Polymenidou M, Cleveland DW. Non-cell autonomous toxicity in neurodegenerative disorders: ALS and beyond. J Cell Biol 2009; 187: 761-772.

61. Marchetto MC, Muotri AR, Mu Y, Smith AM, Cezar GG, Gage FH. Non-cell-autonomous effect of human SOD1 G37R astrocytes on motor neurons derived from human embryonic stem cells. Cell Stem Cell 2008; 3: 649-657.

62. Heuer E, Rosen RF, Cintron A, Walker LC. Nonhuman primate models of Alzheimer-like cerebral proteopathy. Curr Pharm Des 2012; 18: 1159-1169.

63. Muratore CR, Srikanth P, Callahan DG, Young-Pearse TL. Comparison and optimization of hiPSC forebrain cortical differentiation protocols. PLoS One 2014; 9: e105807.

64. Brennand KJ, Simone A, Jou J, Gelboin-Burkhart C, Tran N, Sangar S et al. Modelling schizophrenia using human induced pluripotent stem cells. Nature 2011; 473: 221-225.

Supplementary Information accompanies this paper on Cell Death and Differentiation website (http://www.nature.com/cdd) 